\title{
Ab initio Ultrafast Spin Dynamics in Solids
}

\author{
Junqing Xu, ${ }^{1}$ Adela Habib, ${ }^{2}$ Ravishankar Sundararaman, ${ }^{3}$ and Yuan Ping ${ }^{1}$ \\ ${ }^{1}$ Department of Chemistry and Biochemistry, University of California, Santa Cruz, CA 95064, USA \\ ${ }^{2}$ Department of Physics, Applied Physics and Astronomy, \\ Rensselaer Polytechnic Institute, 110 8th Street, Troy, New York 12180, USA \\ ${ }^{3}$ Department of Materials Science and Engineering, \\ Rensselaer Polytechnic Institute, 110 8th Street, Troy, New York 12180, USA
}

(Dated: October 1, 2021)

\begin{abstract}
Spin relaxation and decoherence is at the heart of spintronics and spin-based quantum information science. Currently, theoretical approaches that can accurately predict spin relaxation of general solids including necessary scattering pathways and capable for ns to ms simulation time are urgently needed. We present a first-principles real-time density-matrix approach based on Lindblad dynamics to simulate ultrafast spin dynamics for general solid-state systems. Through the complete first-principles descriptions of pump, probe and scattering processes including electronphonon, electron-impurity and electron-electron scatterings with self-consistent electronic spin-orbit couplings, our method can directly simulate the ultrafast pump-probe measurements for coupled spin and electron dynamics over ns at any temperatures and doping levels. We first apply this method to a prototypical system GaAs and obtain excellent agreement with experiments. We found that the relative contributions of different scattering mechanisms and phonon modes differ considerably between spin and carrier relaxation processes. In sharp contrast to previous work based on model Hamiltonians, we point out that the electron-electron scattering is negligible at room temperature but becomes dominant at low temperatures for spin relaxation in n-type GaAs. We further examine ultrafast dynamics in novel spin-valleytronic materials - monolayer and bilayer $\mathrm{WSe}_{2}$ with realistic defects. We find that spin relaxation is highly sensitive to local symmetry and chemical bonds around defects. For the bilayer $\mathrm{WSe}_{2}$, we identify the scattering pathways in ultrafast dynamics and determine relevant dynamical properties, essential to its utilization of unique spin-valley-layer locking effects. Our work provides a predictive computational platform for spin dynamics in solids, which has unprecedented potentials for designing new materials ideal for spintronics and quantum information technology.
\end{abstract}

\section{INTRODUCTION}

Spin is a fundamental quantum mechanical property of electrons and other particles. The spin states can be used as the basis of quantum bits in quantum information science (QIS) ${ }^{1}$, in addition to being used in spintronics analogous to electrical charge in conventional electronics ${ }^{2}$. The key property for spintronics and spin-based QIS is the lifetime of spin states. Stable manipulations of spin states in practical applications require lifetimes on the order of hundreds of nanoseconds or even milliseconds. Determining the underlying mechanisms and controlling spin relaxation are vital to reach long spin lifetimes at room temperature. Experimentally spin relaxation can be studied through ultrafast magneto-optical pump-probe ${ }^{\sqrt[314]{4}}$ and spin transport measurements ${ }^{5}$, allowing the direct observations of dynamical processes and quantitative determination of spin relaxation time, $\tau_{s}$.

Despite significant experimental progresses and several proposed systems in the past decades ${ }^{216}$, materials with properties required for practical QIS and spintronics applications such as long $\tau_{s}$ at room temperature remain to be found ${ }^{11517}$. Theoretical predictions of materials properties have been mostly focused on electronic excitations $^{8} 10$ and electron-hole recombinations $\frac{11}{13}$ of potential spin defects for QIS applications. Reliable prediction of spin lifetime and dominant relaxation mechanism will allow rational design of materials in order to accelerate the identification of ideal materials for quantum technologies, while forgoing the need of experimental search over a large number of materials.

Until recently, most state-of-the-art theoretical methods to study spin dynamics of solid-state materials are limited to simplified and system-specific models that require prior input parameters214 16. These methods laid important theoretical foundation for spin dynamics, such as the spin-Bloch kinetic equations developed from Nonequilibrium Green's Function theory (NEGFT) 17 . Ref. 18 derived a closed equation of motion for the electronic single-particle density matrix, including different scattering matrices, which may be applicable to spin dynamics. However, because of the simplified electronic structure and electron-phonon coupling matrices, quantitative prediction of spin relaxation remains out of reach. Occasionally, even trends in $\tau_{s}$ predicted by such models may be incorrect as shown recently for graphene ${ }^{[19}$ Furthermore, these models are unable to provide predictive values for new materials where prior inputs are not available.

Prior to our work, the existing first-principles methodology for spin lifetime has been mostly based on spin-flip matrix elements in a specialized Fermi's Golden rule ${ }^{20} 22$, which is only applicable to systems with Kramers' degeneracy or spatial inversion symmetry, not suitable to lots of materials promising for quantum computing and spintronics applications 215 . Other first-principles techniques like real-time Time-Dependent Density Functional The- 
ory (TDDFT) ${ }^{23}$ are challenging for crystalline systems due to high computational cost for describing phonon relaxations that require large supercells. More importantly, long simulation time over nanoseconds often required by spin relaxation is a major difficulty for TDDFT, which is only practical for tens to a few hundred femtoseconds. While spin dynamics based on TDDFT has been recently performed for ultrafast demagnetization of magnetic systems within tens of $\mathrm{fs}_{\mathrm{s}}{ }^{24} 26$, the intrinsic time scale and supercell limitations mentioned above remain.

We recently derived a generalized rate equation ${ }^{27}$ based on first-principles density matrix (DM) Lindblad dynamics framework, which provides accurate spin relaxation time due to spin-orbit and electron-phonon couplings for a broad range of materials, with arbitrary symmetry. However, our previous work requires the system is already at a quasi-equilibrium state when the dynamics can be described by a single-exponential decay, which can not describe coupled spin and carrier dynamics at an out-of-equilibrium state in ultrafast pump-probe experiments. In this work, we develop a real-time $a b$ initio DM dynamics method based on this theoretical framework, with complete descriptions of scattering processes including electron-phonon (e-ph), electron-impurity (e-i), and electron-electron (e-e), being adequate for over ns to ms simulation time. Specifically, compared to the generalized rate equation in our previous work, DM dynamics with explicit real-time evolutions allow coupled carrier and spin relaxation away from quasi-equilibrium with all decoherence pathways simultaneously. This will facilitate direct prediction of experimental signatures in ultrafast magneto-optical spectroscopy to unambiguously interpret experimental probes of spin and electron dynamics.

We will demonstrate the generality of our approach by considering two prototypical and disparate systems GaAs and few-layer $\mathrm{WSe}_{2}$, which have very different spin relaxation mechanisms.

We will first apply our DM dynamics methodology to investigate ultrafast spin dynamics of GaAs, which has broad interest in spintronics over past decades 228 and more recently $\sqrt[32]{34}$, partly due to its long spin lifetime especially in the n-doped material at relatively low temperature ${ }^{28}$. Despite various experimenta $\left[\begin{array}{l}28 / 29 / 35 \\ 37\end{array}\right.$ and theoretical 2|30|31|38 40 (mostly using parameterized model Hamiltonian) studies previously, the dominant spin relaxation mechanism of bulk GaAs under various temperatures and doping levels remains unclear. For example, Refs. 30 and 39 claimed e-i and e-ph scatterings dominate spin relaxation at low and room temperatures, respectively; however, Refs. 31 and 40 conclude that e-e may be more important at room temperature and even more at lower temperatures. Moreover, electron-phonon scattering matrices which can be accurately obtained from first-principles, are very difficult to be precisely described in parameterized models used previously. Most importantly, the applicability of empirical D'yakonovPerel' (DP) relation, which is widely used for describing inversion-asymmetric systems including GaAs, needs to be carefully examined. Throughout this work, we provide complete and unambiguous insights on the underlying mechanism of spin relaxation and applicability of the DP relation for GaAs from first-principles DM dynamics.

Due to broken inversion symmetry and strong SOC, monolayer transition metal dichalcogenides (TMDs) exhibit exciting physical properties including valley-specific optical excitation and spin-valley locking effects. In Ref. 4142, it has been shown that by introducing doping in monolayer TMDs, ultraslow decays of Kerr rotations, which correspond to ultralong spin/valley lifetimes of resident carriers especially resident holes can be observed at low temperatures. Those features make monolayer TMDs advantageous for spin-valleytronics and (quantum) information processing.

Besides monolayers, bilayer TMDs recovering inversion symmetry have also attracted significant interests because of the new "layer" degree of freedom or layer pseudospin in addition to spin and valley pseudospin 43,45 . Previous studies already concluded that electronic states at $K / K^{\prime}$ valleys of a bilayer TMD are approximately a superposition of those of two monolayers. This allows us to tune which layer carriers/spins are localized by a perpendicular electric field $E_{z}$, and make use of the spinvalley-layer locking effects for spin-valleytronic applications.

Although spin/valley relaxation of resident carriers in monolayer TMDs, which is most relevant to spin-valleytronic applications, have been extensively examined $\sqrt{27 / 41 / 42 / 46}$, the underlying dynamics especially the effects of different types of impurities have not been investigated through predictive $a b$ initio simulations. Furthermore, for bilayer TMDs, the study on spin/valley dynamics is still in infancy with few ultrafast measurements which however do not exhibit long spin relaxation time and are lack of spin-valley-layer locking property 47 49. There is a lack of knowledge of the role of scattering processes and the scattering pathways for spin/valley dynamics of free carriers in bilayers, which prevents researchers to realize and manipulate spin-valley-layer locking effects for designing spinvalleytronic devices.

In this work, we will answer the above questions by performing $a b$ initio real-time dynamics simulations with a circularly polarized pump pulse and relevant scattering mechanisms. We focus on $\mathrm{WSe}_{2}$ due to its larger valence band SOC splitting and focus on dynamics of holes since $\tau_{s}$ of holes seem longer than electrons.

In the following, we first introduce our theoretical formalism of real-time density-matrix approach with various scattering processes and pump-probe spectroscopy. In particular, we focus on spin-orbit mediated spin relaxation and decoherence processes under the existences of electron scatterings, which are rather common in semiconductors and metals2117. We use this method to simulate pump-probe Kerr rotation and real-time spin dynamics, by using GaAs as a prototypical example and 
comparing with experiments. Next, we study spin lifetime dependence on the temperature and doping level, where the dominant mechanisms can vary significantly. We then discuss the roles of different scattering mechanisms and phonon modes in carrier and spin relaxations, respectively, in order to resolve related long-standing controversies. We further simulate ultrafast dynamics in monolayer and bilayer $\mathrm{WSe}_{2}$ and extract useful dynamical properties. Our work provides predictive theory and computational platform for open quantum dynamics, and offers new and critical insights for spin relaxation and decoherence in general solid-state systems.

\section{THEORY}

\section{A. Real-time density-matrix dynamics and spin relaxation time}

To provide a general formulation of quantum dynamics in solid-state materials, we start from the Liouville-von Neumann equation in the interaction picture,

$$
\begin{aligned}
& \frac{d \rho(t)}{d t}=-i\left[H^{\prime}(t), \rho(t)\right], \\
& H^{\prime}(t)=H(t)-H_{0}(t),
\end{aligned}
$$

where $H, H_{0}$ and $H^{\prime}$ are total, unperturbed and perturbed Hamiltonian, respectively. In this work, the total Hamiltonian is

$$
\begin{aligned}
H & =H_{0}+H_{\text {pump }}+H_{\mathrm{e}-\mathrm{i}}+H_{\mathrm{e}-\mathrm{ph}}+H_{\mathrm{e}-\mathrm{e}}, \\
H_{0} & =H_{e, 0}+H_{\text {efield }}+H_{z}+H_{\mathrm{ph}},
\end{aligned}
$$

where $H_{e, 0}$ is electronic Hamiltonian under zero external field. In this work, $H_{\text {efield }}$ is Hamiltonian induced by a perpendicular electric field $E_{z}$ along the vacuum direction. $H_{z}$ is the Zeeman Hamiltonian corresponding to an external magnetic field $\mathbf{B}, H_{z}=g_{s} \mu_{B} \mathbf{B} \cdot \mathbf{s}$, where $\mathbf{s}=\left(s_{x}, s_{y}, s_{z}\right)$ and $s_{i}$ is spin matrix in Bloch basis under zero field. $g_{s}$ is $g$ factor and $\mu_{B}$ is the Bohr magneton. $H_{\text {pump }}$ is the Hamiltonian of the pump pulse and will be described below. $H_{\mathrm{ph}}$ is the phonon Hamiltonian, while $H_{\mathrm{e}-\mathrm{i}}, H_{\mathrm{e}-\mathrm{ph}}$ and $H_{\mathrm{e}-\mathrm{e}}$ describe the electronimpurity, electron-phonon and electron-electron interactions respectively. The detailed forms of the interaction Hamiltonians are given in Appendix A.

In practice, the many-body density matrix master equation in Eq. 1 is reduced to a single-particle one and the environmental degrees of freedom are traced out 50 . The total rate of change of the density matrix is separated into terms related to different parts of Hamiltonian,

$$
\frac{d \rho}{d t}=\left.\frac{d \rho}{d t}\right|_{\mathrm{coh}}+\left.\frac{d \rho}{d t}\right|_{\text {scatt }},
$$

where $\rho$ is the density matrix of electrons. Above, $\left.\frac{d \rho}{d t}\right|_{\text {coh }}$ describes the coherent dynamics of electrons under potentials or fields, e.g. the applied pump pulse, while $\left.\frac{d \rho}{d t}\right|_{\text {scatt }}$ captures the scattering between electrons and other particles.

To obtain Eq. 5 which involves only the dynamics of electrons or the electronic subsystem, we have assumed the environmental subsystem is not perturbed by the change of the electronic subsystem, which in this work means there is no dynamics of phonons. This assumption is valid when the system is not far from equilibrium, e.g., when excitation is weak. In most spin dynamics experiments, it is desirable to work in the low excitation density limit to avoid additional complexities and focus on the physics of spin dynamics. Indeed in many experiments, e.g., in Refs. 28 and 51, pump fluence and excitation density are controlled to be low, e.g., excitation density $2 \times 10^{14} \mathrm{~cm}^{-2}$ for GaAs. Therefore, phonon dynamics can be safely excluded in the current stage. The inclusion of phonon degrees of freedom in the densitymatrix dynamics has been discussed in detail in Refs. 5052 with model Hamiltonian, which can be our future work to implement from first-principles.

To define spin lifetime, we follow the time evolution of the observable

$$
S_{i}=\operatorname{Tr}\left(s_{i} \rho\right)
$$

where $s_{i}$ is the spin operator $(i=x, y, z)$. This time evolution must start at an initial state (at $t=t_{0}$ ) with a net spin i.e. $\delta \rho\left(t_{0}\right)=\rho\left(t_{0}\right)-\rho^{\text {eq }} \neq 0$ such that $\delta S_{i}\left(t_{0}\right)=S_{i}\left(t_{0}\right)-S_{i}^{\text {eq }} \neq 0$, where "eq" corresponds to the final equilibrium state. We evolve the density matrix through Eq. 5 using an adaptive Runge-Kutta fourthorder method for a long enough simulation time, typically from tens of ps to several ns, until the evolution of $S_{i}(t)$ can be reliably fitted by

$$
\begin{aligned}
S_{i}(t)-S_{i}^{\mathrm{eq}}= & {\left[S_{i}\left(t_{0}\right)-S_{i}^{\mathrm{eq}}\right] \exp \left[-\frac{t-t_{0}}{\tau_{s, i}}\right] } \\
& \times \cos \left[\omega_{B}\left(t-t_{0}\right)+\phi\right] .
\end{aligned}
$$

to extract the relaxation time, $\tau_{s, i}$. Above, $\omega_{B}$ is oscillation frequency due to energy splitting in general, which under an applied magnetic field $\mathbf{B}$ would include a contribution $\approx 0.5 g_{s} \mu_{B}\left(\mathbf{B} \times \hat{\mathbf{S}_{\mathbf{i}}}\right)$.

In order to examine whether the spin relaxation time depends on how the spin imbalance is generated, we implement two general ways to initialize $\delta \rho\left(t_{0}\right)$. First, for simulating pump-probe experiments, we choose $\delta \rho\left(t_{0}\right)$ corresponding to interaction with a pump pulse. Second, we use the technique proposed previously in Ref. 27 by applying a test magnetic field at $t=-\infty$, allowing the system to equilibrate with a net spin and then turning it off suddenly at $t_{0}$. 


\section{B. Scattering terms}

The scattering part of the master equation can be separated into contributions from several scattering channels,

$$
\left.\frac{d \rho}{d t}\right|_{\text {scatt }}=\left.\sum_{c} \frac{d \rho}{d t}\right|_{c}
$$

where $c$ labels a scattering channel. Under Born-Markov approximation, in general we have ${ }^{18}$

$$
\left.\frac{d \rho_{12}}{d t}\right|_{c}=\frac{1}{2} \sum_{345}\left[\begin{array}{c}
(I-\rho)_{13} P_{32,45}^{c} \rho_{45} \\
-(I-\rho)_{45} P_{45,13}^{c, *} \rho_{32}
\end{array}\right]+\text { H.C., }
$$

where $P^{c}$ is the generalized scattering-rate matrix and H.C. is Hermitian conjugate. The subindex, e.g., "1", is the combined index of $\mathrm{k}$-point and band. The weights of $\mathrm{k}$ points must be considered when doing sum over $\mathrm{k}$ points. Note that $P^{c}$ in the interaction picture is related to its value $P^{S, c}$ in the Schrodinger picture as

$$
P_{1234}^{c}(t)=P_{1234}^{S, c} \exp \left[i\left(\epsilon_{1}-\epsilon_{2}-\epsilon_{3}+\epsilon_{4}\right) t\right],
$$

where $\epsilon_{i}$ are single-particle eigenvalues of $H_{0}$. Below, we consider three separate scattering mechanisms - electron-impurity (e-i), electron-phonon (e-ph) and electron-electron (e-e), and describe the matrix elements for each.

For electron-phonon scattering, the scattering matrix is given by 18

$$
\begin{aligned}
P_{1234}^{\mathrm{S}, \mathrm{e}-\mathrm{ph}} & =\sum_{q \lambda \pm} A_{13}^{q \lambda \pm} A_{24}^{q \lambda \pm, *} \\
A_{13}^{q \lambda \pm} & =\sqrt{\frac{2 \pi}{\hbar}} g_{12}^{q \lambda \pm} \sqrt{\delta_{\sigma}^{G}\left(\epsilon_{1}-\epsilon_{2} \pm \omega_{q \lambda}\right)} \sqrt{n_{q \lambda}^{ \pm}}
\end{aligned}
$$

where $q$ and $\lambda$ are phonon wavevector and mode, $g^{q \lambda \pm}$ is the electron-phonon matrix element, resulting from the absorption $(-)$ or emission $(+)$ of a phonon, computed with self-consistent spin-orbit coupling from firstprinciples, ${ }^{53} n_{q \lambda}^{ \pm}=n_{q \lambda}+0.5 \pm 0.5$ in terms of phonon Bose factors $n_{q \lambda}$, and $\delta_{\sigma}^{G}$ represents an energy conserving $\delta$-function broadened to a Gaussian of width $\sigma$.

Next, for electron-impurity scattering, the scattering matrix is given by

$$
\begin{aligned}
P_{1234}^{\mathrm{S}, \mathrm{e}-\mathrm{i}} & =A_{13}^{i} A_{24}^{i, *}, \\
A_{13}^{i} & =\sqrt{\frac{2 \pi}{\hbar}} g_{13}^{i} \sqrt{\delta_{\sigma}^{G}\left(\epsilon_{1}-\epsilon_{3}\right)} \sqrt{n_{i} V_{\text {cell }}}, \\
g_{13}^{i} & =\left\langle 1\left|V^{i}\right| 3\right\rangle,
\end{aligned}
$$

where $n_{i}$ and $V_{\text {cell }}$ are impurity density and unit cell volume, respectively, and $V^{i}$ is the impurity potential. In this work, we deal with ionized and neutral impurities differently. For ionized impurities, $V^{i}$ is proportional to screened Coulomb potential $\sqrt{54}$; for neutral impurities, we compute impurity potentials with supercell methods from DFT. (See Appendix A for further details).

Finally, for electron-electron scattering, the scattering matrix is given by 18

$$
\begin{aligned}
P_{12,34}^{\mathrm{S}, \mathrm{e}-\mathrm{e}} & =2 \sum_{56,78}(I-\rho)_{65} \mathscr{A}_{15,37} \mathscr{A}_{26,48}^{*} \rho_{78} \\
\mathscr{A}_{1234} & =\frac{1}{2}\left(A_{1234}-A_{1243}\right) \\
A_{1234} & =\frac{1}{2} \sqrt{\frac{2 \pi}{\hbar}}\left[g_{1234}^{e-e}\left(\delta_{\sigma, 1234}^{G}\right)^{1 / 2}+g_{2143}^{e-e}\left(\delta_{\sigma, 2143}^{G}\right)^{1 / 2}\right] \\
g_{1234}^{\mathrm{e}-\mathrm{e}} & =\left\langle 1(r)\left|\left\langle 2\left(r^{\prime}\right)\left|V\left(r-r^{\prime}\right)\right| 3(r)\right\rangle\right| 4\left(r^{\prime}\right)\right\rangle
\end{aligned}
$$

where $V\left(r-r^{\prime}\right)$ is the screened Coulomb potential and $\delta_{\sigma, 1234}^{G}=\delta_{\sigma}^{G}\left(\epsilon_{1}+\epsilon_{2}-\epsilon_{3}-\epsilon_{4}\right)$ is a Gaussian-broadened energy conservation function. The screening is described by Random-Phase-Approximation (RPA) dielectric function (details in Appendix A). Although the above equations describe all possible scattering processes between electrons and holes, we only consider those between conduction electrons here, which are appropriate for ntype Group III-V semiconductors 30139. The electronhole scattering can be important for intrinsic and p-type

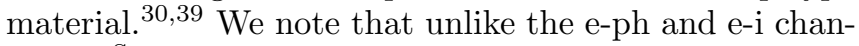
nels, $P^{\mathrm{S} \text {,e-e }}$ (as well as the dielectric screening in $V$ ) is a function of $\rho$ and needs to be updated during time evolution of $\rho$. This is a clear consequence of the two-particle nature of e-e scattering. $P^{\mathrm{S} \text {,e-e }}$ can be written as the difference between a direct term and an exchange term,

$$
\begin{aligned}
P^{\mathrm{S}, \mathrm{e}-\mathrm{e}} & =P^{\mathrm{S}, \mathrm{e}-\mathrm{e}, \mathrm{d}}-P^{\mathrm{S}, \mathrm{e}-\mathrm{e}, \mathrm{x}}, \\
P^{\mathrm{S}, \mathrm{e}-\mathrm{e}, \mathrm{d}} & =\sum_{56,78}(I-\rho)_{65} A_{15,37} A_{26,48}^{*} \rho_{78}, \\
P^{\mathrm{S}, \mathrm{e}-\mathrm{e}, \mathrm{x}} & =\sum_{56,78}(I-\rho)_{65} A_{15,37} A_{26,84}^{*} \rho_{78} .
\end{aligned}
$$

According to Ref. [50, the direct term is expected to dominate the dynamical scattering processes between conduction or valence electrons, allowing us to neglect the exchange term here.

\section{Pump-probe simulation}

In nonrelativistic limit, the light-matter interaction Hamiltonian operator $\left(\widehat{H}_{e-p}\right)$ reads 55

$$
\begin{aligned}
\widehat{H}_{e-p}= & \frac{e}{m_{e}} \mathbf{A}(t) \cdot \widehat{\mathbf{p}} \\
& +\frac{e}{2 m_{e}} \mathbf{A}(t) \cdot \mathbf{A}(t)+g_{e} \mu_{B} \widehat{\mathbf{s}} \cdot(\nabla \times \mathbf{A}(t)),
\end{aligned}
$$

where $\mathbf{A}(t)$ is the vector potential and $\mathbf{A}(t)=$ $\mathbf{A}_{0}(t) e^{-i \omega t}+\mathbf{A}_{0}^{*}(t) e^{i \omega t}$ with $\mathbf{A}_{0}(t)$ being the complex amplitude and $\omega$ being photon frequency. $\widehat{\mathbf{p}}$ is momentum operator. $g_{e} \approx 2.0023192$ is anomalous gyromagnetic ratio. The second quadratic term plays a 
role only when pump fluence is higher by several orders of magnitude than that in usual spin dynamics experiments and can be safely neglected. Since $\nabla \times \mathbf{A}(t)=$ $-i \mathbf{q}_{\text {photon }} \times \mathbf{A}(t)^{\sqrt[55]{5}}$ and the photon wavevector $\mathbf{q}_{\text {photon }}$ is quite small (the photon wavelength is much longer than the scale of unit cells), the third term is also negligible. Therefore, we will only keep the first $\mathbf{A}(t) \cdot \widehat{\mathbf{p}}$ term.

The interaction with a pump pulse of frequency $\omega_{\text {pump }}$ in the interaction picture is given by

$$
\begin{aligned}
H_{\text {pump }, k, m n}\left(\omega_{\text {pump }}, t\right)= & \frac{e}{m_{e}} \mathbf{A}_{0}(t) \cdot \mathbf{p}_{k, m n} e^{i t\left(\epsilon_{m}-\epsilon_{n}-\omega_{\text {pump }}\right)} \\
& + \text { H.C. }
\end{aligned}
$$

where $m, n$ represent the band indices and $k$ represents the $\mathrm{k}$ point sampling in the first Brillouin zone. For a Gaussian pulse centered at time $t_{\text {center }}$ with width $\tau_{\text {pump }}$,

$\mathbf{A}_{0}(t)=\mathbf{A}_{0} \frac{1}{\sqrt{\sqrt{\pi} \tau_{\text {pump }}}} \exp \left[-\left(t-t_{\text {center }}\right)^{2} /\left(2 \tau_{\text {pump }}^{2}\right)\right]$.

Note that the corresponding pump fluence is $I_{\text {pump }}=$ $\omega_{\text {pump }}^{2}\left|A_{0}\right|^{2} /(8 \pi \alpha)$, where $\alpha$ is fine structure constant. As a part of the coherent portion of the time evolution, the dynamics due to this term are captured directly in the Liouville form,

$$
\left.\frac{d \rho}{d t}\right|_{\text {pump }}=-i\left[H_{\text {pump }}, \rho\right] .
$$

The probe pulse interacts with the material similarly to the pump pulse, and could be described in exactly the same way in principle. However, this would require repeating the simulation for several values of the pumpprobe delay. Instead, since the probe is typically chosen to be of sufficiently low intensity, we use second-order time-dependent perturbation theory to capture its interaction with the system,

$\Delta \rho^{\text {probe }}=\frac{1}{2} \sum_{345}\left\{\begin{array}{c}{[I-\rho(t)]_{13} P_{32,45}^{\text {probe }} \rho(t)_{45}} \\ -[I-\rho(t)]_{45} P_{45,13}^{\text {probe }, *} \rho(t)_{32}\end{array}\right\}+H . C$.

where $P^{\text {probe }}$ is the generalized scattering-rate matrix for the probe in the interaction picture. Its corresponding Schrodinger-picture quantity is

$$
\begin{aligned}
P_{1234}^{\mathrm{S}, \text { probe }} & =\sum_{ \pm} A_{13}^{\text {probe }, \pm} A_{24}^{\text {probe }, \pm, *} \\
A_{13}^{\text {probe }, \pm} & =\sqrt{\frac{2 \pi}{\hbar}} \frac{e}{m_{e}}\left(\mathbf{A}_{0}^{\text {probe }} \cdot \mathbf{p}\right) \sqrt{\delta_{\sigma}^{G}\left(\epsilon_{1}-\epsilon_{3} \pm \omega_{\text {probe }}\right)}
\end{aligned}
$$

The dielectric function change $\Delta \epsilon$ between the excited state and ground state absorption detected by the probe is then

$$
\operatorname{Im} \Delta \epsilon=\frac{2 \pi}{\left(\omega_{\text {probe }}\right)^{3}\left|A_{0}^{\text {probe }}\right|^{2}} \operatorname{Tr}\left(H_{0} \Delta \rho^{\text {probe }}\right) .
$$

Note that $\Delta \rho^{\text {probe }}$ contains $\left|A_{0}^{\text {probe }}\right|^{2}$ so that $\operatorname{Im} \Delta \epsilon$ is independent of $A_{0}^{\text {probe }}$. The above $\operatorname{Im} \Delta \epsilon$ is a functional of the density matrix according to Eq. 26 and is an extension of the usual independent-particle Im $\epsilon$ depending on just occupation numbers $\sqrt[58]{[5}$ After computing $\operatorname{Im} \Delta \epsilon$ above, the real part $\operatorname{Re} \Delta \epsilon$ can be obtained from the Krames-Kronig relation.

By summing up the dielectric function change $\Delta \epsilon$ computed above with the dielectric function for ground state absorption, we can obtain the excited-state $\epsilon$ as inputs for Kerr and Faraday rotation calculations. $\underline{59}$ These correspond to the rotations of the polarization plane of a linearly polarized light, reflected by (Kerr) and transmitted through (Faraday) the material, after a pump excitation with a circularly-polarized light. Time-Resolved Kerr/Faraday Rotation (TRKR/TRFR) has been widely used to study spin dynamics of materials $28 / 36$. In a TRKR experiment, a circularly-polarized pump pulse is used to excite valence electrons of the sample to conduction bands. The transitions approximately satisfy the selection rule of $\Delta m_{j}= \pm 1$ for left and right circularlypolarized pulses, respectively, where $m_{j}$ is secondary total angular momentum. TRKR works by measuring the changes of polarization of reflected light, which qualitatively is proportional to the small population imbalance of electronic states with different $m_{j}$.

Specifically, the Kerr rotation angle $\theta_{K}$ is computed with dielectric functions by

$$
\theta_{K}=\operatorname{Im} \frac{\sqrt{\epsilon_{+}}-\sqrt{\epsilon_{-}}}{1-\sqrt{\epsilon_{+}} \sqrt{\epsilon_{-}}}
$$

where \pm denotes the left and right circular polarization, respectively.

\section{COMPUTATIONAL DETAILS}

The ground-state electronic structure, phonon, and eph matrix element calculations of GaAs and few-layer $\mathrm{WSe}_{2}$ are first calculated using Density Functional Theory (DFT) with relatively coarse $k$ and $q$ meshes in the JDFTx plane-wave DFT code ${ }^{60}$ For GaAs, we use the experimental lattice constant of $5.653 \AA, 61$ and select the SCAN exchange-correlation functional ${ }^{62}$ for an accurate description of the electron effective mass (see section II in Supplemental Materials ${ }^{63}$ ). We also apply a scissor operator to the DFT values to reach experimental band gap $1.43 \mathrm{eV}^{64}$. For $\mathrm{WSe}_{2}$, we used PBE exchange correlation functional along with the DFT-D2 pair potential dispersion corrections 5 . The resulting lattice constant is $3.32 \AA$ and distance between two $\mathrm{W}$-atom planes is $6.419 \AA$ close to experimental values of bulk $\mathrm{WSe}_{2}$, 3.297 and $6.491 \AA$. The phonon calculations of GaAs and $\mathrm{WSe}_{2}$ employ a $4 \times 4 \times 4$ and $6 \times 6$ supercell, respectively. We use Optimized Norm-Conserving Vanderbilt (ONCV) pseudopotentials 67 with self-consistent spin-orbit coupling throughout, which we find converged 
at a plane-wave kinetic energy cutoff of 34 and 62 Ry for GaAs and $\mathrm{WSe}_{2}$, respectively. With these computational parameters, we find the effective mass of conduction electrons of GaAs to be $0.054 m_{e}$, close to the experimental value of $0.067 m_{e}$. 64 . (More convergence tests can be found in Supporting Information (SI) $\left.{ }^{63}\right)$.

We then transform all quantities from plane wave basis to maximally localized Wannier function basis ${ }^{68}$, and interpolate them $53[69] 73$ to substantially finer $\mathrm{k}$ and $\mathrm{q}$ meshes. The Wannier interpolation approach fully accounts for polar terms in the e-ph matrix elements and phonon dispersion relations, using the approach developed by Verdi and Giustind ${ }^{74}$ for 3D and using the methods in Ref. 75 and Ref. 76 for 2D systems. The Born effective charges and dielectric constants are calculated from open-source code QuantumESPRESSO 77.

For GaAs, the fine $k$ and $q$ meshes are $288 \times 288 \times 288$ for simulations at $300 \mathrm{~K}$ and are finer at lower temperature, e.g., $792 \times 792 \times 792$ for simulations at $30 \mathrm{~K}$. This is necessary to sample enough electronic states around band edges and for spin lifetime convergence within $20 \%$. The $k$ and $q$ convergence are easier for $\mathrm{WSe}_{2}$ due to much larger effective masses and we used $168 \times 168$ and $600 \times 600$ meshes at 50 and $10 \mathrm{~K}$, respectively. The computation of e-i and e-e matrix elements and the real-time dynamics simulations are done with a new custom code interfaced to JDFTx. The energy-conservation smearing parameter $\sigma$ is chosen to be comparable or smaller than $k_{B} T$ for each calculation. Detailed convergence tests of number of $\mathrm{k}$ points and energy window for electronic states at various smearing parameters can be found in Supplemental Materials 63 .

\section{RESULTS AND DISCUSSIONS}

\section{A. Applications to n-doped GaAs}

\section{Spin dynamics and its relation to TRKR}

In general, time evolution of Kerr rotation angle $\theta_{K}$ (see Eq. 30) is not equivalent to that of spin along the direction of reflected light, and in fact, they can be quite different in some cases ${ }^{41}$. There are few first-principles studies of TRKR considering scattering processes in a form of semiclassical Boltzmann equation $\frac{58}{}$. A full quantum description of scatterings with non-diagonal density matrix in TRKR has not been presented in previous firstprinciples studies, to the best of our knowledge. And the relation between dynamics of $\theta_{K}$ and spin observable for general systems including GaAs has not yet been well examined.

Using our density-matrix approach, we are able to directly simulate the nonequilibrium ultrafast dynamics of optically excited systems during which the dynamics of different electronic quantities such as spin and carriers can be strongly coupled. We include all scattering terms in a full quantum description as shown in the theory sec-
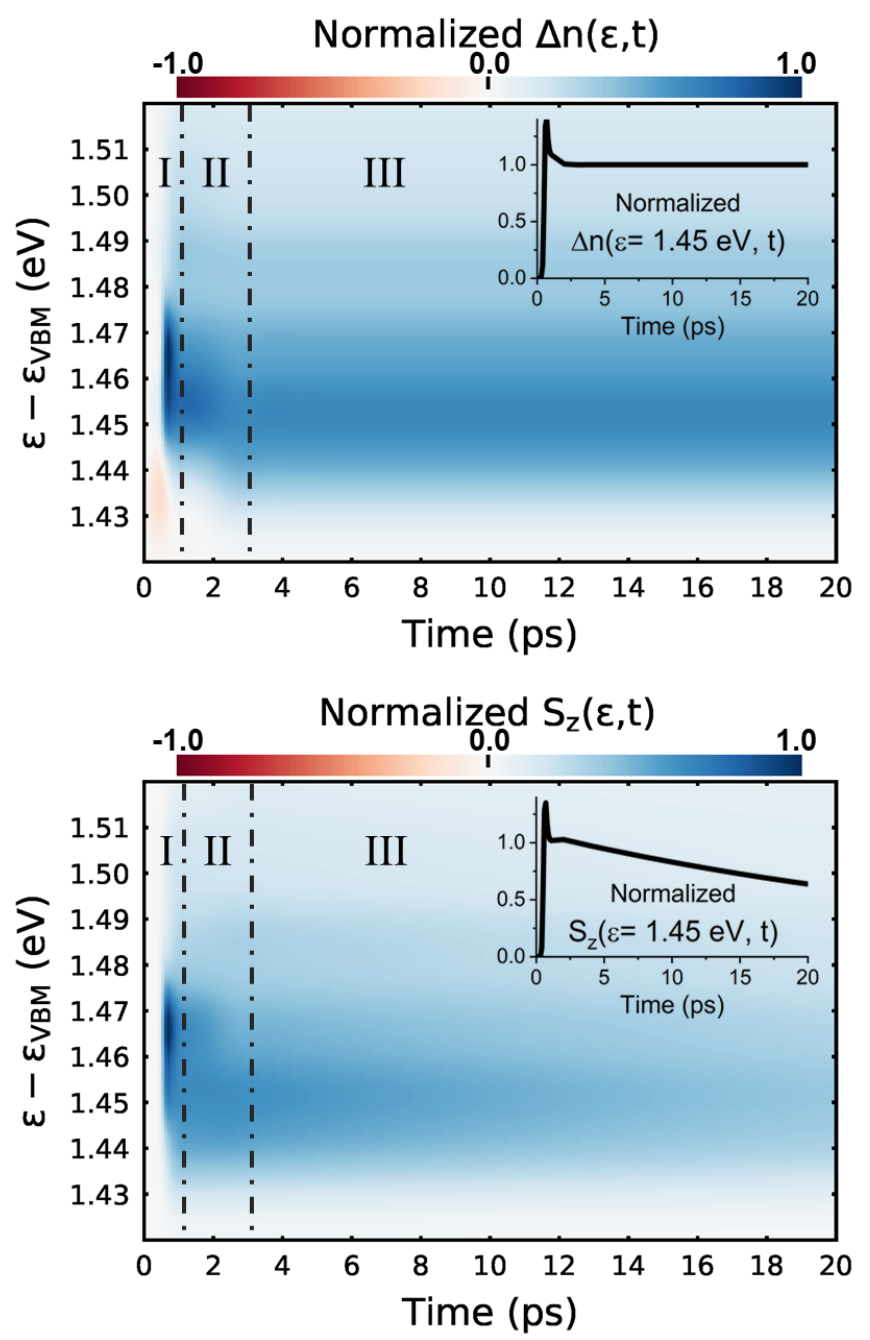

FIG. 1. The energy-resolved dynamics of carriers (a) $\Delta n(\epsilon, t)=n(\epsilon, t)-n(\epsilon, 0)$ and (b) spins $S_{z}(\epsilon, t)$ of conduction electrons with a circularly polarized pump pulse centered at $0.5 \mathrm{ps}$. The insets on the top right of both panels show $\Delta n(\epsilon, t)$ and $S_{z}(\epsilon, t)$ at $\epsilon=1.45 \mathrm{eV}$. The pump energy $\omega_{\text {pump }}=1.47 \mathrm{eV}$ is chosen to be higher than band gap 1.43 $\mathrm{eV}^{64}$. The width of the pump pulse $\tau_{\text {pump }}$ is $100 \mathrm{fs}$. The pump fluence $I_{\text {pump }}$ is low at $0.01 \mu \mathrm{J} \mathrm{cm}^{-2}$. The dynamics can be approximately divided into three regions - Region I, II and III labeled in this figure. In Region I, the system is excited by a pump pulse. In Region II, pump processes are already finished, then both carriers and spins relax simultaneously. In Region III, carrier distribution stays unchanged while spins keep decaying.

tion II.B and Appendix A. We perform the real-time dynamics simulations of n-type GaAs for tens of ps at room temperature and several ns at low temperature until the fitted spin lifetime does not change any more. Having temporal density matrix, we can further analyze the dynamics of various observables, including occupation, spin and Kerr rotation angle easily. We then examine the relation between $\theta_{K}$ and spin in the dynamics.

Figure 1 shows the energy-resolved dynamics of car- 
(a)

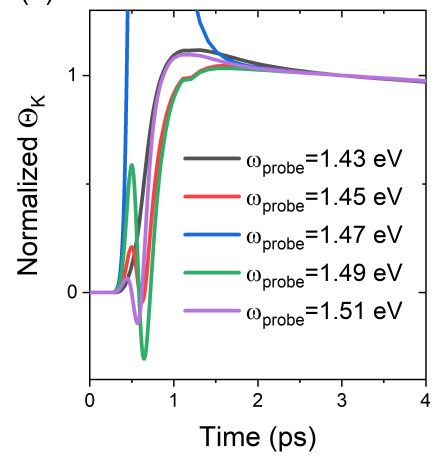

(b)

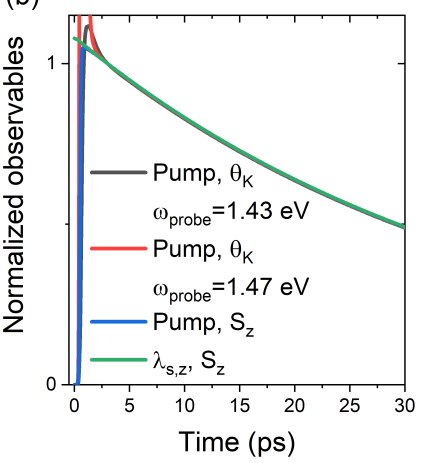

FIG. 2. (a) Compare the dynamics of Kerr rotation angle $\theta_{K}$ at different probe energies $\omega_{\text {probe }}$ excited by a circularly polarized pump pulse in first few ps. Fast oscillations in first 2 ps are due to the pump pulse and coupled spin and carrier relaxation. (b) Compare relaxation of different observables - $\theta_{K}$ with different $\omega_{\text {probe }}$ (denoted by black and red lines) and $S_{z}$ with initial spin imbalance generated by a pump pule (Pump) or a test magnetic field along $\mathrm{z}$ direction $\lambda_{s, z} \sim 0.001-0.1$ Tesla (blue and green lines). $\omega_{\text {pump }}=1.47 \mathrm{eV}$. The pump pulse is centered at $0.5 \mathrm{ps}$. The longer time-scale dynamics (over 10 ps) for $\theta_{K}$ and $S_{z}$ has similar relaxation time independent on the generation method of spin imbalance and specific probe energies.

riers $\Delta n(\epsilon, t)=n(\epsilon, t)-n(\epsilon, 0)$ and spins $S_{z}(\epsilon, t)$. The energy-resolved observable $O(\epsilon)$ is defined as $\operatorname{Re}\left[\sum_{k, m n} o_{k, m n} \rho_{k, n m} \delta\left(\epsilon-\epsilon_{k m}\right)\right]$, where $o$ is operator matrix. We can see that during the first ps (region I in Fig. 1), both observables vary quickly due to the existence of the pump processes and both have their maximum at an energy slightly lower than the pump energy, $1.47 \mathrm{eV}$ (slightly larger than the band gap $1.43 \mathrm{eV}$ ), at a time shortly after the time center of the pump pulse 0.5 ps. Interestingly, after pump being not active or after $0.8-1 \mathrm{ps}$, carriers and spins simultaneously relax until 2-3 ps (region II in Fig. 1 1 and 1p). Afterward (region III in Fig. 1 a and 1b), carriers stay unchanged but spins $S_{z}(\epsilon, t)$ decay exponentially as shown in the insets of Fig. $1 \mathrm{a}$ and $1 \mathrm{~b}$.

We have further analyzed the dynamics of Kerr rotation angle $\theta_{K}$ and compared it with spin dynamics. From Fig. 22a, we can see that during pump processes and shortly after them (from 0 to $2 \mathrm{ps}$ ), $\theta_{K}(t)$ has strong oscillations and sensitive to the probe energy $\omega_{\text {probe }}$. The $\omega_{\text {probe-sensitivity may be partly attributed to the energy }}$ dependence of carrier and spin dynamics. From Fig. $2 \mathrm{a}$ and $2 \mathrm{~b}$, it can be seen that after 3 ps (or in time region III defined in Fig. 11, $\theta_{K}$ with different $\omega_{\text {probe }}$ decay exactly the same. We can also find that with a pump pulse, relaxation time of the Kerr rotation is the same as that of $S_{z}$, i.e. $\tau_{s, z}$. Moreover, it turns out that $\tau_{s, z}$ does not depend on how spin imbalance is generated - by a circularly polarized pump pulse or by turning off a test magnetic field along $z$ direction (see Sec. II A). This may indicate that if the system is not extremely far from equilibrium,

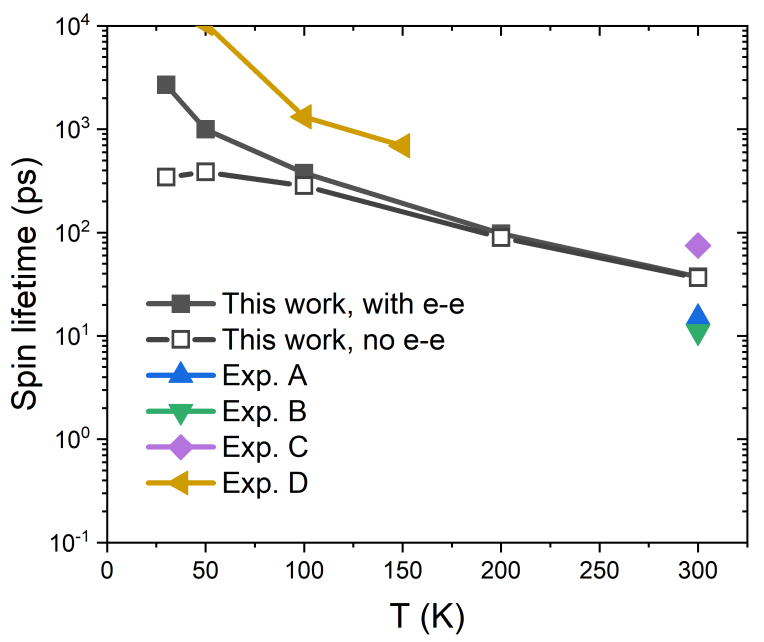

FIG. 3. Theoretical spin lifetime with (black solid square) and without (black empty square) the electron-electron scattering compared with experimental data. Exp. A, B, C and D are experimental data from Refs. $36,37 / 78$ and 28 , respectively.

spin relaxation along direction $i$ is not sensitive to the way of generating spin imbalance, as long as the degrees of freedom other than $S_{i}$ are not relevant or disappear in a short time. According to these observations, hereinafter, we will do real-time dynamics starting from a $\delta \rho$ generated by turning off a test magnetic field and fit $\tau_{s, z}$ from time evolution of $S_{z}$.

We have also studied the effects of $\omega_{\text {pump }}$ and pump fluence $I_{\text {pump }}$ on spin relaxation of $n$-GaAs at $300 \mathrm{~K}$. We find that $\omega_{\text {pump }}$ has very weak effects on spin relaxation but $\tau_{s, z}$ decreases with pump fluence. See more details in Appendix C.

\section{Temperature-dependence of spin lifetime and its dominant relaxation mechanism}

As discussed earlier, long-standing controversies remain for the dominant spin relaxation mechanism of GaAs at different temperature and doping leve 3 which will be resolved in the following sections. We start from study $\tau_{s, z}$ of $n$-GaAs as a function of temperature at a moderate doping level $\left(2 \times 10^{16} \mathrm{~cm}^{-3}\right)$. For simplicity, we assume all impurities are fully ionized, so that the impurity density $n_{i}$ is equal to the free carrier density $n_{\text {free }}$. We first compared our calculated spin lifetime with experimental results in Fig. 3. Our results of $\tau_{s, z}$ of $n$-GaAs give good agreement with experiments at various temperatures $28 / 36 / 37 / 78$. Different experiments have slight variations between each other due to sample preparation conditions and specific measurement techniques. The spin lifetime increases from tens of ps at room temperature to tens of ns at low temperature. Note that e-e scattering plays an essential role at low temperatures, i.e. by comparing with (black solid square) and without (black empty square) in Fig. 3. The correct temperature 

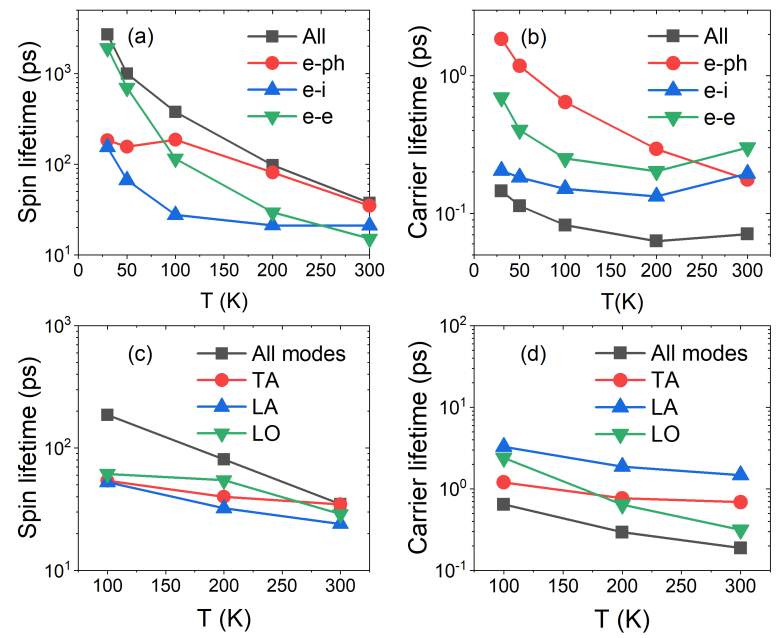

FIG. 4. Spin and carrier lifetimes of $n$-GaAs with $n_{i}=$ $2 \times 10^{16} \mathrm{~cm}^{-3}$ with different scattering mechanisms and different phonon modes. In (a) and (b), "All" represents all the e-ph, e-i and e-e scattering mechanisms being considered. TA, LA and LO represent transverse acoustic, longitudinal acoustic and longitudinal optical modes, respectively. The carrier lifetimes $\bar{\tau}_{p}$ present are the inverse of averaged carrier scattering rates $\left\langle\tau_{p}^{-1}\right\rangle$. The method of carrier lifetime calculations is given in Appendix B. \langle\rangle means taking average around chemical potential $\mu$. For a state-resolved quantity $A_{k n}$, its average is defined as $\langle A\rangle=\sum_{k n} A_{k n}\left[f^{\text {eq }}\right]^{\prime}\left(\epsilon_{k n}\right) / \sum_{k n}\left[f^{\text {eq }}\right]^{\prime}\left(\epsilon_{k n}\right)$, where $\left[f^{\text {eq }}\right]^{\prime}$ is the derivative of Fermi-Dirac function.

dependence of $\tau_{s, z}$ can be reproduced only if e-e scattering is included.

We further examine the contributions of different scattering mechanisms to carrier and spin lifetime respectively, as a function of temperature. Different from spin lifetime obtained from real-time DM dynamics including all scattering processes simultaneously, the carrier lifetime $\left(\bar{\tau}_{p}\right)$ is defined through the inverse of the averaged carrier scattering $\operatorname{rate}\left(\tau_{p}^{-1}\right): \bar{\tau}_{p}=1 /\left\langle\tau_{p}^{-1}\right\rangle$. Various scattering processes (e-e, e-i and e-ph) contribute to the total carrier scattering rates through $\tau_{p}^{-1}=\left(\tau_{p}^{\mathrm{e}-\mathrm{e}}\right)^{-1}+\left(\tau_{p}^{\mathrm{e}-\mathrm{i}}\right)^{-1}+\left(\tau_{p}^{\mathrm{e}-\mathrm{ph}}\right)^{-1} .\langle\rangle$ means taking average around chemical potential $\mu$. For a stateresolved quantity $A_{k n}$, its average is defined as $\langle A\rangle=$ $\sum_{k n} A_{k n}\left[f^{\mathrm{eq}}\right]^{\prime}\left(\epsilon_{k n}\right) / \sum_{k n}\left[f^{\mathrm{eq}}\right]^{\prime}\left(\epsilon_{k n}\right)$, where $\left[f^{\mathrm{eq}}\right]^{\prime}$ is the derivative of Fermi-Dirac function. For both carrier and spin lifetime, the lifetime due to the most dominant scattering channel is the closest to the one including all processes (black squares in both Fig. 4a and b). For spin relaxation in Fig. 44 , at low temperature below $50 \mathrm{~K}$, e-e scattering is the most dominant process as discussed above. However, the e-ph process becomes more dominant above 100K. On the other hand, for carrier relaxation in Fig. $4 \mathrm{p}$, the e-i process is dominant over a wide temperature range from low to right below room temperature. At room temperature, for both spin and carrier lifetimes, the e-ph scattering is the most important process (closest to the total lifetime with all scattering processes).

Our observations differ from those in Refs. 31 and 40 , where the authors also found that e-e scattering dominates spin relaxation at lower temperatures, e.g. $77 \mathrm{~K}$, but their results showed that at room temperature ee scattering can be more important than other scatterings and enhances $\tau_{s, z}$ of $n$-GaAs by about $100 \%$ with moderate doping concentrations. The overestimate of the effects of e-e scattering at room temperature is most likely a limitation of the semiclassical method employed therein.

Similarly, we also find that different phonon modes can play different roles in carrier and spin relaxations as shown in Fig. 纬 and 4 d. For example, at room temperature, LO (longitudinal optical) mode is most important for carrier relaxation but seems less important than TA (transverse acoustic) modes for spin relaxation. The situation is the opposite at $100 \mathrm{~K}$ where TA/LO is most important for carrier/spin relaxation. Our finding that TA modes are slightly more important than LO mode in spin relaxation at room temperature is different from what have been believed in previous model studies $30 / 40$, where they declared that the electron-LO-phonon scattering dominates spin relaxation at high temperatures especially at room temperature. This disparity is most likely due to differences in the e-ph matrix elements and electronic quantities, where we used fully first-principles approaches instead of parameterized models in previous work.

In addition, we find the total spin lifetime is the longest when considering all scattering processes in Fig. $4 \mathrm{p}$; in contrast, the carrier lifetime is the shortest including all scattering mechanism in Fig. 4 $\mathrm{b}$. This follows the inverse relation between spin and carrier lifetime in the empirical D'yakonov-Perel' (DP) mechanism ${ }^{2114}$ for systems without inversion symmetry, as will be discussed in more details in next section.

\section{Doping-level-dependence of spin lifetime and its dominant relaxation mechanism}

Figure 5 shows the carrier and spin lifetimes with different doping density $n_{i}$ at $30 \mathrm{~K}$ with individual and total scattering pathways, respectively. Similar to temperature dependence and phonon contributions, it is also found that the roles of different scattering mechanism differ considerably between spin and carrier relaxation processes. Specifically, for the carrier relaxation in Fig. 5b, except when $n_{i}$ is very low (e.g. at $10^{14} \mathrm{~cm}^{-3}$ ), the electron-impurity scattering (e-i) dominates, similar to the case of carrier lifetime over a large range of temperature at a moderate doping in Fig. 4p. On the other hand, for the spin relaxation in Fig. 5 , the e-e scattering dominates except at very high concentration (above $10^{17} \mathrm{~cm}^{-3}$ ), while e-i scattering is only important in the very high doping region (close to or above $10^{17} \mathrm{~cm}^{-3}$ ).

Figure 5 shows the calculated $\tau_{s}$ has a maximum at 

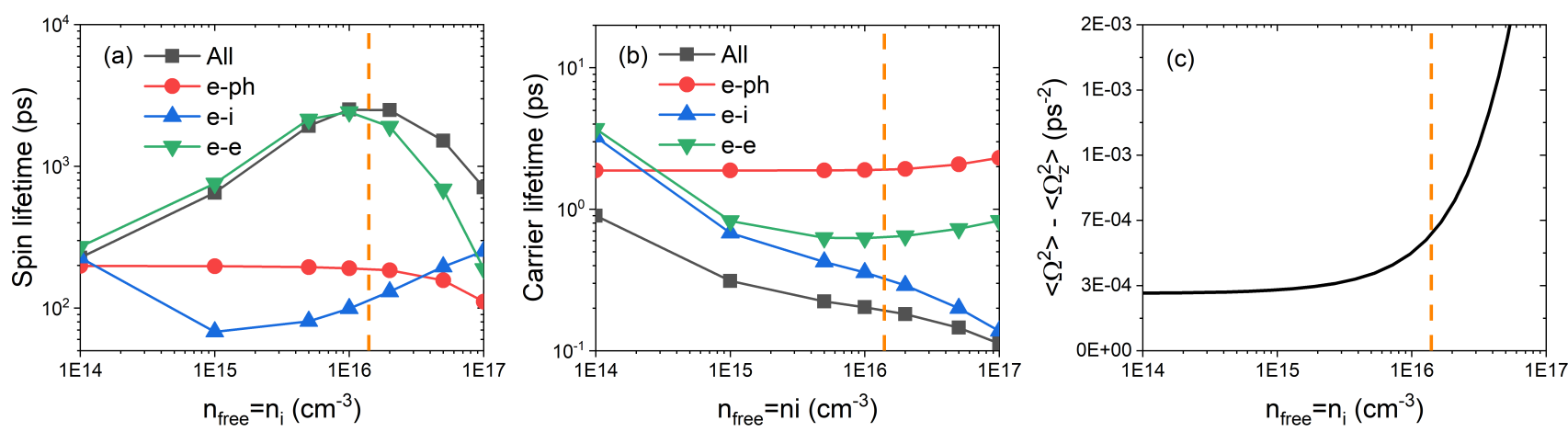

FIG. 5. (a) Spin and (b) carrier lifetimes of $n$-GaAs with different doping concentrations at $30 \mathrm{~K}$ with different scattering mechanisms. "All" represents all the e-ph, e-i and e-e scattering mechanisms being considered. (c) $\left\langle\boldsymbol{\Omega}^{2}\right\rangle-\left\langle\Omega_{i}^{2}\right\rangle$ as a function of carrier density, where $\boldsymbol{\Omega}$ is the Larmor frequency due to the "internal" magnetic field computed from first-principles, which describes the SOC term induced by inversion asymmetry.

$n_{i}=1-2 \times 10^{16} \mathrm{~cm}^{-3}$, and $\tau_{s}$ decreases fast with $n_{i}$ going away from its peak position. This is in good agreement with the experimental finding in Ref. 28, which also reported $\tau_{s}$ at $n_{i}=10^{16} \mathrm{~cm}^{-3}$ is longer than $\tau_{s}$ at other lower and higher $n_{i}$ at a low temperature (a few Kelvin). The $n_{i}$ dependence of $\tau_{s}$ may be qualitatively interpreted from the commonly used empirical DP relation ${ }^{2}$ for inversion-asymmetric systems, $\tau_{s, i} \sim \tau_{s, i}^{\mathrm{DP}}=$ $1 /\left[\bar{\tau}_{p} \cdot\left(\left\langle\boldsymbol{\Omega}^{2}\right\rangle-\left\langle\Omega_{i}^{2}\right\rangle\right)\right]$, where $\bar{\tau}_{p}$ is the carrier lifetime, and $\boldsymbol{\Omega}$ is the Larmor frequency due to the "internal" magnetic field, which describes the SOC term induced by inversion asymmetry. For spin $1 / 2$ systems, the internal magnetic field at $\mathbf{k}\left(\boldsymbol{\Omega}_{\mathbf{k}}\right)$ will induce an energy splitting $\Delta_{\mathrm{k}}$ and polarize the spin along the direction of $\boldsymbol{\Omega}_{\mathrm{k}}$. Previously, $\boldsymbol{\Omega}_{\mathbf{k}}$ was mostly obtained with model Hamiltonian with Dresselhaus SOC field ${ }^{79}$, which is rather qualitative. Instead, we obtained k-dependent internal magnetic field $\boldsymbol{\Omega}_{\mathrm{k}}$ from first-principles calculations, by using $\Omega_{\mathbf{k}, i}=2 \Delta_{\mathbf{k}} \cdot s_{\mathbf{k}, i}^{\exp } / \hbar$, where $s_{\mathbf{k}, i}^{\exp }$ is the spin expectation value.

From Fig. 5. we find that with $n_{i}$ from $10^{14} \mathrm{~cm}^{-3}$ to $5 \times 10^{15} \mathrm{~cm}^{-3}$, carrier lifetime $\bar{\tau}_{p}$ decreases rapidly (black curve in Fig. 5b) and $\left\langle\Omega^{2}\right\rangle-\left\langle\Omega_{i}^{2}\right\rangle$ remains flat in Fig. 5;, which may explain why spin lifetime $\left(\tau_{s}\right)$ increases in Fig. 5 a based on the DP relation; however, when $n_{i}>10^{16} \mathrm{~cm}^{-3}, \bar{\tau}_{p}$ decreases with a similar speed but $\left\langle\Omega^{2}\right\rangle-\left\langle\Omega_{i}^{2}\right\rangle$ experiences a sharp increase, which may explain why spin lifetime decreases in Fig. 5 b and owns a maximum at $10^{16} \mathrm{~cm}^{-3}$.

Note that although the above empirical DP relation is intuitive to understand the cause of doping-level dependence of spin lifetime, it may break down when we evaluate individual scattering processes. For example, when $n_{i}$ increases from $10^{14} \mathrm{~cm}^{-3}$ to $10^{15} \mathrm{~cm}^{-3}$, both carrier lifetime $\bar{\tau}_{p}$ and spin lifetime $\tau_{s, z}$ due to e-i scattering decrease while the internal magnetic field remains unchanged. Moreover, the simple empirical relation cannot possibly explain our first-principles results that the e-e and e-i scatterings have largely different contributions in carrier and spin relaxation. First-principles calculations are critical to provide unbiased mechanistic insights to spin and carrier relaxation of general systems.

\section{B. Applications to few-layer $\mathrm{WSe}_{2}$}

\section{Spin/valley relaxation of resident holes of monolayer $W \mathrm{We}_{2}$}

For holes of monolayer $\mathrm{WSe}_{2}$, spin/valley relaxation is mostly determined by intervalley spin-flip scattering processes between $K$ and $K^{\prime}$ valleys because of the spinvalley locking. Previously, we reported spin/valley lifetimes of resident holes of monolayer TMDs at $\mathrm{T} \geq 50 \mathrm{~K}$ with e-ph scattering 27 . At very low temperatures, e.g., 10 $\mathrm{K}$, intervalley e-ph scattering is however not activated as the corresponding phonon occupation is negligible; therefore, other scattering mechanisms are necessary to be included. Note that e-e scattering should not play an important role in spin relaxation of holes of TMDs. The reason is: The e-e scattering is a two-particle process where a transition is accompanied by another transition with energy and momentum being conserved. Considering the fact that only the highest occupied band is involved (see band structure in Fig. S5) in dynamics of TMD holes, for an e-e process, a $K \rightarrow K^{\prime}\left(K^{\prime} \rightarrow K\right)$ spin-flip transition must be accompanied by an opposite $K^{\prime} \rightarrow K\left(K \rightarrow K^{\prime}\right)$ spin-flip transition. Overall, e-e scattering processes have negligible contributions to spin relaxation of TMD semiconductors. As a result, we will include only e-ph and e-i scatterings for $\mathrm{WSe}_{2}$. We use the supercell method to compute e-i scattering matrix elements for neutral defects with self-consistent SOC and more details can be found in Appendix A.

Experimentally several types of impurities/defects exist in TMD samples. Here we pick four types of impurities with different symmetries and chemical bonds (see Fig. 6(a)) - Se vacancy $\left(\mathrm{V}_{\mathrm{Se}}\right)$, two neighboring Se vacancies $\left(\mathrm{V}_{2 \mathrm{Se}-\mathrm{N}}\right), \mathrm{W}$ vacancy $\left(\mathrm{V}_{\mathrm{W}}\right)$ and two Se vacancies with the same in-plane position $\left(\mathrm{V}_{2 \mathrm{Se}-\mathrm{S}}\right)$. As most point 
(a)

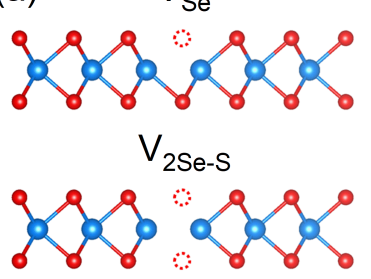

(b) $10^{5}$

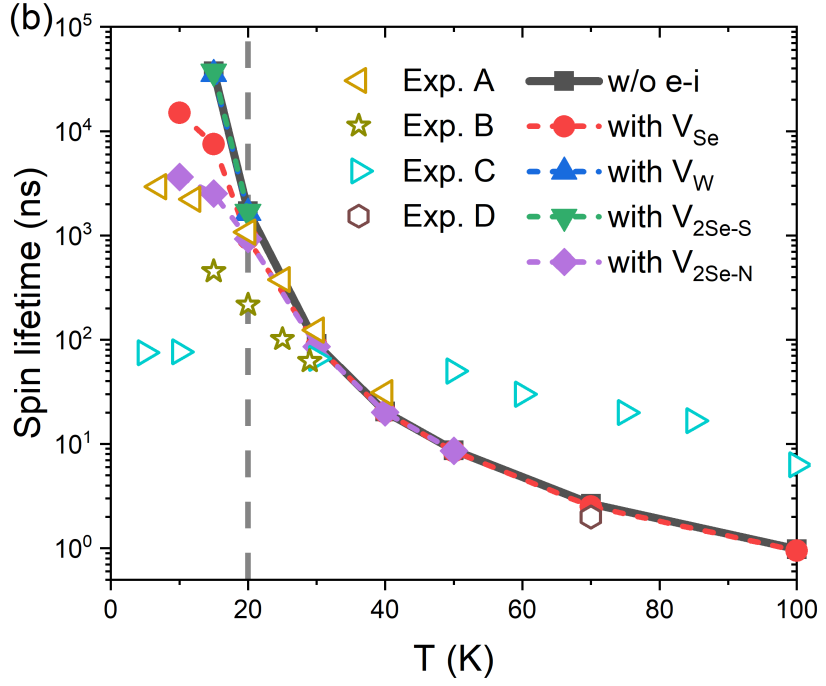

FIG. 6. (a) The schematics of four types of impurities in $\mathrm{WSe}_{2}$. (b) Spin lifetimes of holes of monolayer $\mathrm{WSe}_{2}$ with a relatively low hole density $10^{11} \mathrm{~cm}^{-2}$ with impurities compared with experimental data. Exp. A, B, C and D are experimental data from Refs. 42, 80, 46 and 81, respectively. The choices of impurity concentration $n_{i}$ of different impurities are given in the main text.

defects are relatively deep with large ionization energies in semiconducting TMDs $\$$, we mostly consider neutral defects here. According to Refs. 83 85, the impurity concentration $n_{i}$ ranges from $8 \times 10^{10}$ to $10^{14} \mathrm{~cm}^{-2}$ depending on samples. Considering that $\mathrm{V}_{\mathrm{Se}}$ is often regarded as the most abundant impurity, we choose a reasonable impurity density $n_{i}$ of $\mathrm{V}_{\mathrm{Se}}-7 \times 10^{11} \mathrm{~cm}^{-2}$, within the experimental range and for better comparison with experimental $\tau_{s}$ at $\mathrm{T} \geq 20 \mathrm{~K}$ shown in Fig. 6(b). $n_{i}$ of $\mathrm{V}_{2 \mathrm{Se}-\mathrm{N}}$ is chosen as $8 \times 10^{9} \mathrm{~cm}^{-2}$, two order of magnitude lower than $\mathrm{V}_{\mathrm{Se}}$ because of its larger formation energy 85 and better comparison with experimental $\tau_{s}$. $n_{i}$ of $\mathrm{V}_{\mathrm{W}}$ and $\mathrm{V}_{2 \mathrm{Se}-\mathrm{S}}$ are chosen arbitrarily as we find they have rather weak effects on spin relaxation and are $7 \times 10^{11}$ and $3.5 \times 10^{11} \mathrm{~cm}^{-2}$, respectively.

From Fig. 6, we first find that at $\mathrm{T}>20 \mathrm{~K}$, spin relaxation is almost driven by e-ph scattering and impurities can only affect spin relaxation at $T \leq 20 \mathrm{~K}$. For the effects of different impurities on spin relaxation, we have

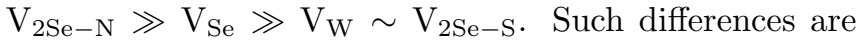
directly related to the large differences among various impurities in electron-impurity matrix elements for the intervalley processes (scattering between $K$ and $K^{\prime}$ valley), i.e. much larger matrix elements $\left|g_{i}\right|$ for interval-
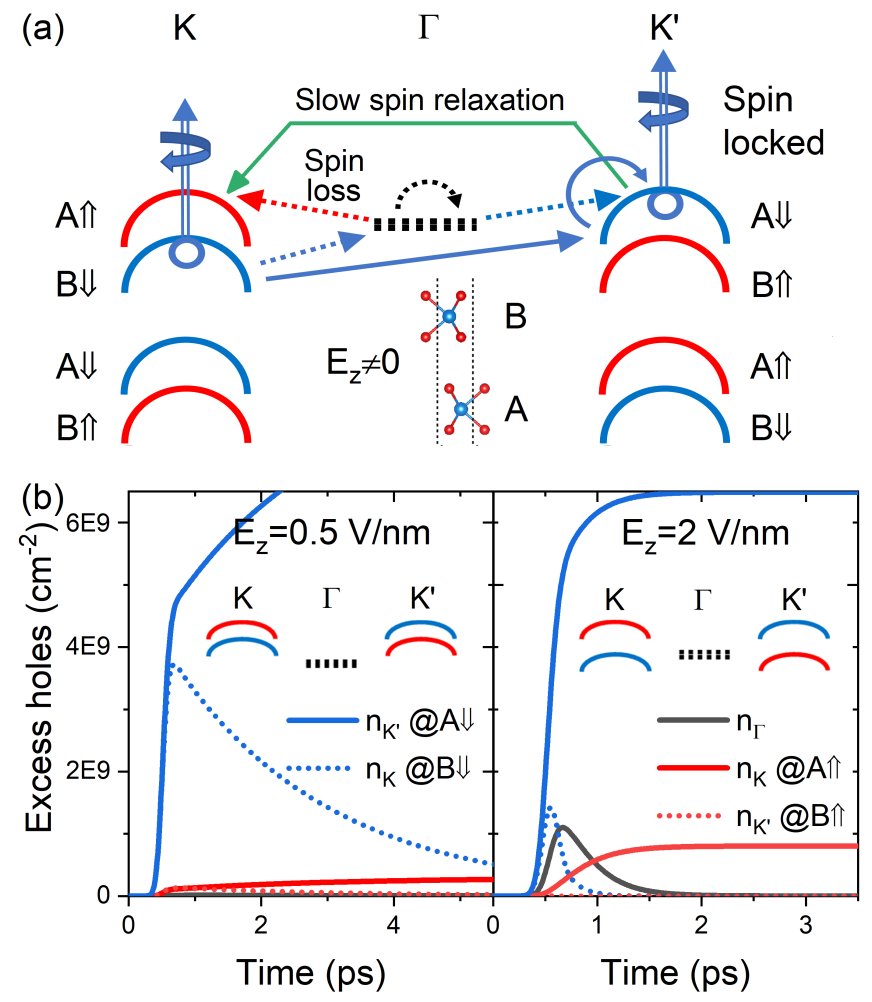

FIG. 7. (a) Schematic diagram of scattering pathways of excited holes in $1 \%$ compressed bilayer $\mathrm{WSe}_{2}$ with a low equilibrium hole density and $1.4 \times 10^{12} \mathrm{~cm}^{-2} \mathrm{~V}_{\text {Se }}$ (double of the monolayer value in Fig. 6) under finite $E_{z}$ during and after a circularly polarized pump, which initially excite holes (labelled as half circles) at both $K$ and $K^{\prime}$ valleys and two top valence bands ("A $\Downarrow$ " at $K^{\prime}$ and "B $\Downarrow$ " at $K$ ). The lower-energy excited holes will decay to the band edge (most to "A $\Downarrow$ " at $K^{\prime}$ and fewer to "A介" at $K$ ) through different scattering pathways. A state being labeled by "A" ("B") means the wavefunction of this state is mostly localized in layer $A(B)$ of the bilayer. $\Uparrow$ and $\Downarrow$ represent spin-up and spin-down, respectively. The color of electronic state represents spin polarization - red and blue mean spin-up and spin-down, respectively. (b) Time evolutions of valley- and band-resolved (excited or excess) hole densities at $50 \mathrm{~K}$ under two $E_{z}$ with a circularly polarized pump. The pump energy is selected to excite electrons at two top valence bands. The pump center is at 0.5 ps. $n_{V}$ represents excess hole density at valley $V$. The insets of panel (b) are the schematics of energies of two top valence bands at $K, K^{\prime}$ and $\Gamma$ valleys under two $E_{z}$ (see calculated band structures in SI Fig. S7).

ley scattering at $\mathrm{V}_{2 \mathrm{Se}-\mathrm{N}}$ and $\mathrm{V}_{\mathrm{Se}}$ compared to the ones at $\mathrm{V}_{\mathrm{W}}$ and $\mathrm{V}_{2 \mathrm{Se}-\mathrm{S}}$ as shown in SI Fig. S9. Moreover, the temperature dependence of $\tau_{s}$ with $\mathrm{V}_{2 \mathrm{Se}-\mathrm{N}}$ is much weaker and in better agreement with experiments than that with $\mathrm{V}_{\mathrm{Se}}$. Therefore, the observed weak temperature dependence in some experiments is probably related to the existence of larger size impurities with lower symmetries (e.g. $\mathrm{V}_{2 \mathrm{Se}-\mathrm{N}}$ ). Our observations suggest that the local symmetry and chemical bonds surrounding an impurity have large impact on spin relaxation. 
Additionally, we have simulated spin lifetimes of monolayer $\mathrm{WSe}_{2}$ at $15 \mathrm{~K}$ with different hole densities and find a strong hole density dependence. The related results are shown in SI Fig. S12.

\section{Ultrafast dynamics of holes of bilayer $W_{S} e_{2}$}

Understanding detailed dynamical processes and related scattering mechanism can help develop strategy of controlling and manipulating spin/valley relaxation through tuning external fields, materials composition, and strain. In the following, we will first identify the scattering pathways of excited holes and spins in bilayer $\mathrm{WSe}_{2}$ (AB stacking as shown in Fig. $7 \mathrm{a}$ ). Next through real-time simulations, we determine dynamical quantities like spin lifetimes and valley polarization at different external fields and strain. Finally, we show the carrier occupation on each layer is fully spin polarized and can be switched by an external electric field.

According to previous studies ${ }^{86 / 87}$ and our calculations, for valence bands of unstrained bilayer $\mathrm{WSe}_{2}, \Gamma$ valley is slightly higher than $K / K^{\prime}$ valley, which is usually undesirable. The $K / K^{\prime}$ valley can be pushed higher than $\Gamma$ valley by applying slight in-plane compressive strain (corresponding band structures can be found in SI Fig. S6). Moreover, under zero $E_{z}$, the bilayer $\mathrm{WSe}_{2}$ has inversion symmetry, leading to carriers being equally populated at $K$ and $K^{\prime}$ valleys and at two layers, with spin up and down degeneracy. A finite $E_{z}$ can break inversion symmetry (thus break Kramers degeneracy) and induce non-zero layer polarization or layer population difference. For example, two top valence bands from layer A with $\Uparrow$ (up spin) and layer B with $\Downarrow$ (down spin) are degenerate at $K$ without electric field but split under electric field. Thus each band is associated with a particular spin channel, valley and layer, i.e. spin-valley-layer locking effect. Also by tuning the sign and magnitude of $E_{z}$, we are able to control various physical quantities like layer pseudospin, band splitting energy, etc. Therefore, to ensure spin-valley-layer locking effects being observed, we will study slightly compressed inversion-symmetric bilayer $\mathrm{WSe}_{2}$ under finite $E_{z}$.

Figure 7(a) shows the scattering pathway schematics for hole bands (half circles) during the first few ps of slightly compressed (1\%) bilayer $\mathrm{WSe}_{2}$ excited by a circularly polarized pump pulse under finite $E_{z}$ at $50 \mathrm{~K}$, before exciton recombination processes happen typically at tens of ps timescale at this temperature.88189 Similar to the GaAs case (see Sec. IV A 1), the hole spins will undergo the following processes: optical generation, decay to band edges and at the end slow relaxation. Initially, holes with the same spin polarization are excited at both $K$ and $K^{\prime}$ valleys and two layers equally (e.g. down spin holes generated at $K$ valley and layer B and $K^{\prime}$ valley and layer $\mathrm{A}$ as shown in Fig. 7 (a)). During and after the excitations, lower-energy holes at $K\left(K^{\prime}\right)$ valley will decay to the band edge through two possible scattering
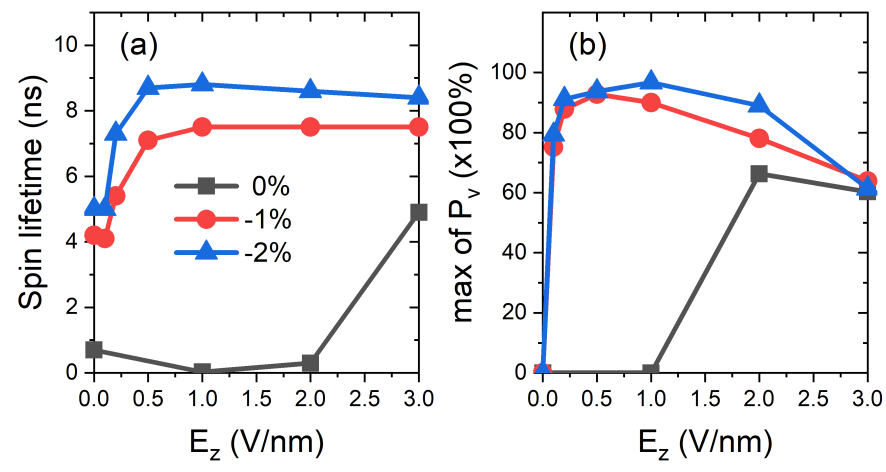

FIG. 8. Spin lifetime and maximum valley polarization $P_{V}=\left|n_{K}-n_{K^{\prime}}\right| / n_{\text {tot }}$ of bilayer $\mathrm{WSe}_{2}$ at $50 \mathrm{~K}$, where $n_{K\left(K^{\prime}\right)}$ is excess hole density at $K\left(K^{\prime}\right)$ valley and $n_{\text {tot }}$ is total excess hole density. Negative strain means compressive strain. $P_{V}$ will be $90 \%$ of its maximum shortly after the pump and reach the maximum at a time from 1 to 20 ps depending on $E_{z}$ and the strain. After reaching its maximum, since most carriers/spins have already decayed to the band edge, $P_{V}$ can relax only very slowly through intervalley spin-flip scattering. This implies that having a high maximum of $P_{V}$ is important to ensure a high $P_{V}$ during a long time.

pathways: (i) Direct pathway through interlayer spinconserving scattering (solid blue line); (ii) Indirect pathway through $\Gamma$-valley-related scattering (dashed lines for both spin conserving and flip processes). After all holes decay to the band edge, most of their carried spins are "locked" at a certain layer and valley (e.g., "A $\Downarrow "$ at $K^{\prime}$ in Fig. 7) due to weak intervalley spin-flip scattering (green arrow in Fig. 7(a)), which is the so-called "spin-valleylayer locking".

In Fig. 7(b), we show time evolutions of valley- and band-resolved (excited or excess) hole densities under two $E_{z}$. It can be seen that under a low $E_{z}(0.5 \mathrm{~V} / \mathrm{nm}$, Fig. 7(b) left panel), the main scattering pathway is the direct one mentioned above, i.e. the spin down holes scattered from $\mathrm{B} \Downarrow$ at $K$ in dashed blue line with decreasing population to $\mathrm{A} \Downarrow$ at $K^{\prime}$ in solid blue line with increasing population. Under a higher $E_{z}(2 \mathrm{~V} / \mathrm{nm}$, Fig. 7 $(\mathrm{b})$ right panel), although the direct scattering pathway still exists, the indirect one through the $\Gamma$ valley also becomes important because the band energy at $\Gamma$ is pushed higher than the second valence band at $K$ and $K^{\prime}$ under this electric field (see inset of Fig. 7(b) right panel). Here occupation at $\mathrm{B} \Downarrow$ at $K$ in dashed blue line rapidly decreased while occupation at $\Gamma$ with both up and down spins in solid black temporarily increased through indirect scattering, and most importantly the $\mathrm{A} \Uparrow$ at $K$ in solid red also increased due to the scattering through $\Gamma$ valley. Increased population at $\mathrm{A} \Uparrow$ at $K$ represents weakening the spinvalley-locking effect. Therefore, the indirect scattering pathway will lead to the reduction of spin density and valley polarization.

We then show two key dynamical quantities - spin lifetime $\tau_{s}$ in Fig. 8(a) and maximum valley polarization of bilayer $\mathrm{WSe}_{2}$ in Fig. 8(b) as a function of $E_{z}$ with 


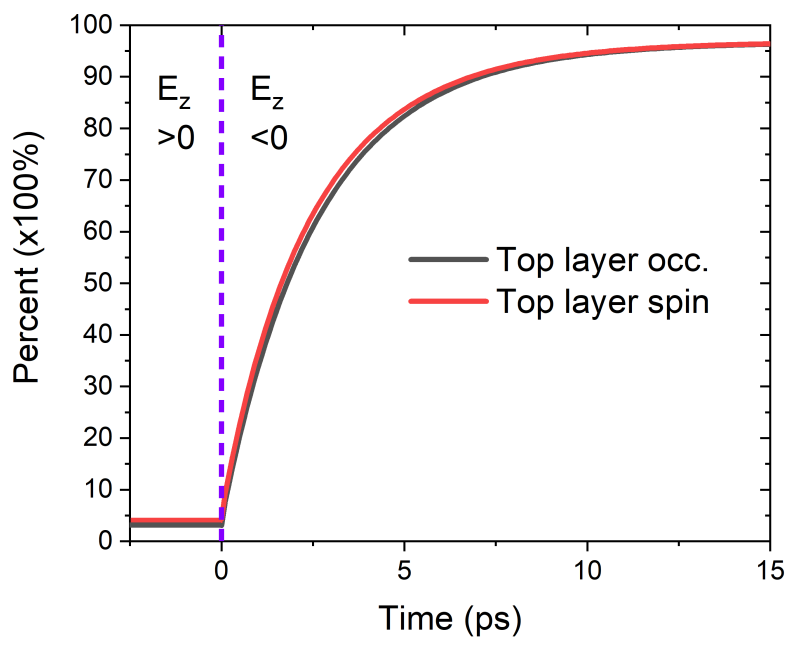

FIG. 9. Time evolution of top layer hole occupation $f_{h}^{\text {top }}$ and spin $s_{z}^{\text {top }}$ (see their definitions in Appendix D) normalized by corresponding total quantities of compressed bilayer $\mathrm{WSe}_{2}$ at $50 \mathrm{~K}$ after the sign of $E_{z}$ is switched at $t=0$. At $t=-10 \mathrm{ps,}$ a pump pulse centered at $t=-9.5 \mathrm{ps}$ with $\tau_{\text {pump }}=100 \mathrm{fs}$ is started to be applied and real-time density matrix dynamics is run under $E_{z}=0.5 \mathrm{~V} / \mathrm{nm}$ until $t=0$ to allow holes/spins to decay to the band edge where states are localized in bottom layer. When the sign of $E_{z}$ is suddenly switched, holes/spins are still localized in the same bottom layer but the new eigenstates around the band edge are localized in top layer, thus holes/spins will transfer from bottom to top layer.

different percentages of strain. Valley polarization is defined as $P_{V}=\left|n_{K}-n_{K^{\prime}}\right| / n_{t o t}$, where $n_{K\left(K^{\prime}\right)}$ is excess hole density at $K\left(K^{\prime}\right)$ valley and $n_{t o t}$ is total excess hole density. A high maximum of $P_{V}$ is necessary to ensure a high $P_{V}$ for extended time. This is because: when $P_{V}$ reaches its maximum, since most carriers/spins have already decayed to the band edge at the same time, $P_{V}$ will decay very slowly through intervalley spin-flip scattering. Obviously, unstrained system is not suitable to utilize spin-valley-layer locking considering its short spin lifetimes and low maximum $P_{V}$ under a relatively low electric field. As we discussed above, a slight strain is helpful to ensure $K / K^{\prime}$ valleys are sufficiently higher than $\Gamma$ valley. Moreover, from Fig. 8, an optimized range of $E_{z}$ is from $\sim 0.2$ to $\sim 2 \mathrm{~V} / \mathrm{nm}$, where the system shows long $\tau_{s}$ and high maximum $P_{V}$, consistent with the fact mentioned above: indirect scattering pathway, which becomes important under high $E_{z}$, will cause loss of spin and valley polarization.

Finally, in a bilayer, the percentage of carriers/spins localized in top/bottom layer at equilibrium can be controlled by a static $E_{z}$. For device applications, it may be desirable to dynamically and spatially tune the locations of holes/spins by controlling $E_{z}$. For this purpose, a knowledge of the speed of carriers/spins transferring between two layers can be useful.

To extract the time scale of such transfer, we first generate holes/spins by applying a circularly polarized pump pulse centered at $-9.5 \mathrm{ps}$ and let the system evolves until $t=0$ under $E_{z}=0.5 \mathrm{~V} / \mathrm{nm}$ to ensure almost all holes/spins being localized in bottom layer, then by switching $E_{z}$ suddenly to $-0.5 \mathrm{~V} / \mathrm{nm}$ at $t=0$, holes/spins will start to transfer to top layer.

In Fig. 9, we show time evolution of top layer hole occupation $f_{h}^{\text {top }}$ and spin $s_{z}^{\text {top }}$ (see their definitions in Appendix D) normalized by corresponding total quantities of $2 \%$ compressed bilayer after the sign of $E_{z}$ is switched. From Fig. 9 at $50 \mathrm{~K}, 90 \%$ switching of $f_{h}^{\text {top }}$ and $s_{z}^{\text {top }}$ takes $\sim 6 \mathrm{ps}$. This time constant is much shorter than $\tau_{s}$, which means such tuning is fast enough to use an electric field as a "switch" in spintronic devices.

\section{CONCLUSIONS}

In this article, we present a first-principles real-time density-matrix approach to simulate ultrafast spin-orbitmediated spin dynamics in solids with arbitrary crystal symmetry. The complete $a b$ initio descriptions of pump, probe and three scattering processes - the electronphonon, electron-impurity and electron-electron scattering in the density-matrix master equation, allows us to directly simulate the nonequilibrium ultrafast pump-probe measurements and makes our method applicable to any temperatures and doping levels. This method has been applied to simulate spin relaxation of $n$-GaAs. We confirm that relaxation time of Kerr rotation and that of spin observables are almost identical and find that relaxation time of spin polarization is relatively robust, i.e. insensitive to how spin imbalance is initialized. Furthermore, we have studied the temperature and doping-level dependencies of spin lifetime and examined the roles of various scattering mechanisms. Overall our theoretical results are in good agreement with experiments. Importantly, our first-principles simulations provide rich mechanistic insights of spin relaxation of $n$-GaAs: we point out that although at low temperatures and moderate doping concentrations e-i scattering dominates carrier relaxation, e-e scattering is the most dominant process in spin relaxation. The relative contributions of phonon modes also vary considerably between spin and carrier relaxation. We have further examined ultrafast dynamics in few-layer $\mathrm{WSe}_{2}$ with realistic impurities. We find that spin relaxation can highly depend on local symmetry and chemical bonds surrounding impurities. For the bilayer, we identify the scattering pathways of holes in ultrafast dynamics and determine relevant dynamical properties, including $\tau_{s}$, maximum valley polarization and layer population/spin switch time, which are essential to utilize its unique spin-valley-layer locking effects. Our method opens up the pathway to predict spin relaxation and decoherence for general materials and provide unbiased insights and guidelines to experimental materials design, which have the potential to revolutionize the field of spintronics and quantum information technologies. 


\section{ACKNOWLEDGEMENTS}

We thank Hiroyuki Takenaka for helpful discussions. This work is supported by National Science Foundation under grant No. DMR-1956015 and the Air Force Office of Scientific Research under AFOSR Award No. FA9550-YR-1-XYZQ. A. H. acknowledges support from the American Association of University Women(AAUW) fellowship program. This research used resources of the Center for Functional Nanomaterials, which is a US DOE Office of Science Facility, and the Scientific Data and Computing center, a component of the Computational Science Initiative, at Brookhaven National Laboratory under Contract No. DE-SC0012704, the lux supercomputer at UC Santa Cruz, funded by NSF MRI grant AST 1828315, the National Energy Research Scientific Computing Center (NERSC) a U.S. Department of Energy Office of Science User Facility operated under Contract No. DE-AC02-05CH11231, the Extreme Science and Engineering Discovery Environment (XSEDE) which is supported by National Science Foundation Grant No. ACI-1548562 20 , and resources at the Center for Computational Innovations at Rensselaer Polytechnic Institute.

\section{APPENDIX A: INTERACTION HAMILTONIAN TERMS AND MATRIX ELEMENTS}

Three interaction Hamiltonian terms in Eq. 3 read

$$
\begin{aligned}
H_{\mathrm{e}-\mathrm{ph}} & =\sum_{12 q \lambda} c_{1}^{\dagger} c_{2}\left(g_{12}^{q \lambda-} b_{q \lambda}+g_{12}^{q \lambda+} b_{q \lambda}^{\dagger}\right) \\
H_{\mathrm{e}-\mathrm{i}} & =n_{i} V_{\text {cell }} \sum_{12} c_{1}^{\dagger} c_{2} g_{12}^{i} \\
H_{\mathrm{e}-\mathrm{e}} & =\sum_{1234} c_{1}^{\dagger} c_{2}^{\dagger} c_{3} c_{4} g_{1234}^{\mathrm{e}-\mathrm{e}} .
\end{aligned}
$$

The e-ph matrix $g^{q \lambda \pm}$ are computed with selfconsistent spin-orbit coupling and Wannier interpolation by the supercell method.

Here we assume impurity density is sufficiently low and the average distance between neighboring impurities is sufficiently long so that the interactions between impurities are negligible. The e-i matrix $g^{i}$ is

$$
\begin{aligned}
g_{13}^{i} & =\left\langle 1\left|\Delta V^{i}\right| 3\right\rangle, \\
\Delta V^{i} & =V^{i}-V^{0}
\end{aligned}
$$

where $V^{i}$ is the potential of the impurity system and $V^{0}$ is the potential of the pristine system. In this work, $g^{i}$ for neutral and ionized impurities are computed differently as follows.

For neutral impurities, $V^{i}$ is computed using a large supercell including an impurity with self-consistent SOC at DFT. This is important for including the detailed potential profile and chemical bonding environment for different impurities. To speed up the supercell convergence, we used the potential alignment method developed in Ref. 91. We checked the supercell size convergence of $g^{i}$ for neutral $\mathrm{V}_{\mathrm{Se}}$ in monolayer $\mathrm{WSe}_{2}$ and found that the corresponding spin lifetime with $6 \times 6$ and $8 \times 8$ supercells differs by only a few percent. Thus $6 \times 6$ supercell is enough for $g^{i}$ of neutral impurities in monolayer $\mathrm{WSe}_{2}$.

For ionized impurities, we use approximate impurity potentials as detailed below. In general, $\Delta V^{i}$ may be separated into two terms $\Delta V^{i}=\Delta V_{\mathrm{ns}}^{i}+\Delta V_{\mathrm{soc}}^{i}$, where $\Delta V_{\mathrm{ns}}^{i}$ is the spin-independent part and $\Delta V_{\mathrm{soc}}^{i}=$ $\left[\hbar /\left(4 m^{2} c^{2}\right)\right] \nabla\left(\Delta V_{\mathrm{ns}}^{i}\right) \times \mathbf{p} \cdot \sigma$ is the SOC correction. For ionized impurities, we approximate $\Delta V_{\mathrm{ns}}^{i}$ as the potential of point charge and is simply the product of the impurity charge $Z$ and the screened Coulomb potential 54 , i.e., $\Delta V_{\mathrm{ns}}^{i}=Z V^{\mathrm{scr}}$. Such approximate describes the long-range part of spin-independent differential impurity potential $\Delta V_{\mathrm{ns}}^{i}$ accurately, which is often the most important contribution from ionized impurities. Considering that $\Delta V_{\mathrm{soc}}^{i}$ is commonly neglected in previous theoretical studies on spin relaxation $\frac{30 \mid 92}{}$ whenever screened Coulomb potential $V^{\mathrm{scr}}$ is used, we will not include $\Delta V_{\mathrm{soc}}^{i}$ for ionized impurities either. Note that such potential for ionized impurities still relaxes spin through spin-mixing and spin-precession. The e-e matrix $g^{\mathrm{e}-\mathrm{e}}$ is

$$
g_{1234}^{\mathrm{e}-\mathrm{e}}=\left\langle 1(r)\left|\left\langle 2\left(r^{\prime}\right)\left|V\left(r-r^{\prime}\right)\right| 3(r)\right\rangle\right| 4\left(r^{\prime}\right)\right\rangle,
$$

where $V\left(r-r^{\prime}\right)$ is the screened electron-electron interaction. The SOC corrections on $V\left(r-r^{\prime}\right)^{93 \mid 94}$ will not be included similar to the ionized impurity case. Thus, $V\left(r-r^{\prime}\right)$ is simply the screened Coulomb potential $V^{s c r}$. Therefore, in both calculations of $g^{i}$ and $g^{\text {e-e }}$, the computation of the screened Coulomb potential $V^{s c r}$ is of key importance.

Currently, we use the static RPA (Random Phase Approximation) dielectric function for the screening and neglect local-field effects. We then show the e-e selfenergy $(\operatorname{Im} \Sigma)$ obtained with such dielectric function well reproduces the one obtained with dynamically screened Coulomb interaction with full RPA dielectric matrix in the relevant energy range as shown in Fig. 10 . The dielectric function has the form

$$
\epsilon(\mathbf{q})=\epsilon_{s} \epsilon^{\text {intra }}(\mathbf{q})
$$

where $\epsilon_{s}$ is the static background dielectric constant and can be calculated by Density Functional Perturbation Theory (DFPT) ${ }^{95}$. $\epsilon^{\text {intra }}(\mathbf{q})$ is the intraband contribution which involves only states with free carriers and is critical for doped semiconductors. It is computed using Random Phase Approximation (RPA),

$$
\epsilon^{\text {intra }}(\mathbf{q})=1-V^{\text {bare }}(\mathbf{q}) \sum_{\mathbf{k} m n}\left(\begin{array}{c}
\frac{f_{\mathbf{k}-\mathbf{q}, m}-f_{\mathbf{k} n}}{\epsilon_{\mathbf{k}-\mathbf{q}, m}-\epsilon_{\mathbf{k}, n}} \times \\
\left|\left\langle u_{\mathbf{k}-\mathbf{q}, m} \mid u_{\mathbf{k} n}\right\rangle\right|^{2}
\end{array}\right) \text {, }
$$

where the sum runs over only states having free carriers, e.g., for a n-doped semiconductor, $m$ and $n$ are 
conduction band indices. In the above formula, $f$ is time-dependent non-equilibrium occupation instead of the equilibrium one $f^{\text {eq }}$. Therefore, if pump is activated or optical field $\mathbf{A}_{0}(t)$ of the pump pulse is not negligible, $\epsilon^{\text {intra }}(\mathbf{q})$ will be updated in every time step, as $f$ will differ from $f^{\text {eq }}$ and the magnitude of difference depends on the excitation density. $V^{\text {bare }}(\mathbf{q})=e^{2} /\left(V_{\text {cell }} \varepsilon_{0}|q|^{2}\right)$ is the bare Coulomb potential with $V_{\text {cell }}$ the unit cell volume and $\varepsilon_{0}$ vacuum permittivity. $u_{\mathbf{k} n}$ is the periodic part of the Bloch wave function.

We then have the matrix elements in reciprocal space,

$$
\begin{aligned}
g_{13}^{i} & =Z V^{\mathrm{scr}}\left(\mathbf{q}_{13}\right)\left\langle u_{1} \mid u_{3}\right\rangle, \\
g_{1234}^{\mathrm{ee}} & =V^{\mathrm{scr}}\left(\mathbf{q}_{13}\right) \delta_{\mathbf{k}_{1}+\mathbf{k}_{2}, \mathbf{k}_{3}+\mathbf{k}_{4}}\left\langle u_{1} \mid u_{3}\right\rangle\left\langle u_{2} \mid u_{4}\right\rangle \\
V^{\mathrm{scr}}\left(\mathbf{q}_{13}\right) & =V^{\text {bare }}\left(\mathbf{q}_{13}\right) / \epsilon\left(\mathbf{q}_{13}\right),
\end{aligned}
$$

where $V^{\text {scr }}(\mathbf{q})$ is the screened Coulomb potential and $\mathbf{q}_{13}=\mathbf{k}_{1}-\mathbf{k}_{3} . \delta_{\mathbf{k}_{1}+\mathbf{k}_{2}, \mathbf{k}_{3}+\mathbf{k}_{4}}$ is Kronecker delta function and means $\mathbf{k}_{1}+\mathbf{k}_{2}=\mathbf{k}_{3}+\mathbf{k}_{4} \cdot\left\langle u_{1} \mid u_{3}\right\rangle$ is the overlap matrix element between two periodic parts of the Bloch wave functions.

\section{APPENDIX B: CARRIER SCATTERING RATE AND IM $\Sigma$ FROM THE DENSITY-MATRIX APPROACH}

At the semiclassical limit, density matrix $\rho$ is replaced by (non-equilibrium) occupation $f$, then the scattering term originally with a full quantum description in Eq. 9 required by DM dynamics becomes:

$$
\left.\frac{d f_{1}}{d t}\right|_{c}=\sum_{2 \neq 1}\left[\left(1-f_{1}\right) P_{11,22}^{c} f_{2}-\left(1-f_{2}\right) P_{22,11}^{c} f_{1}\right],
$$

using the facts that $P_{11,22}$ is real and " $2=1$ " term is zero. "c" represent a scattering channel. Note that the weights of $\mathrm{k}$ points must be considered when doing sum over $\mathrm{k}$ points.

Suppose $f$ is perturbed from its equilibrium value by $\delta f$, i.e., $f=f^{\mathrm{eq}}+\delta f$, then insert $f$ after perturbation into Eq. 42 and linearize it,

$$
\left.\frac{d f_{1}}{d t}\right|_{c}=-\sum_{2 \neq 1}\left[P_{11,22}^{c} f_{2}^{\mathrm{eq}}+\left(1-f_{2}^{\mathrm{eq}}\right) P_{22,11}^{c}\right] \delta f_{1},
$$

using the fact that $\delta P_{11,22}$ is always zero, even for the e-e scattering.

Define carrier relaxation time of state " 1 " $\tau_{p, 1}^{c}$ by $\left.\frac{d f_{1}}{d t}\right|_{c}=-\frac{\delta f_{1}}{\tau_{p, 1}^{c}}$, we have

$$
\frac{1}{\tau_{p, 1}^{c}}=\sum_{2 \neq 1}\left[P_{11,22}^{c} f_{2}^{\mathrm{eq}}+\left(1-f_{2}^{\mathrm{eq}}\right) P_{22,11}^{c}\right]
$$

The linewidth or the imaginary part of the self-energy for the scattering channel $c$ is related to the carrier relaxation time by $\operatorname{Im} \Sigma_{1}^{c}=\hbar /\left(2 \tau_{p, 1}^{c}\right)$.

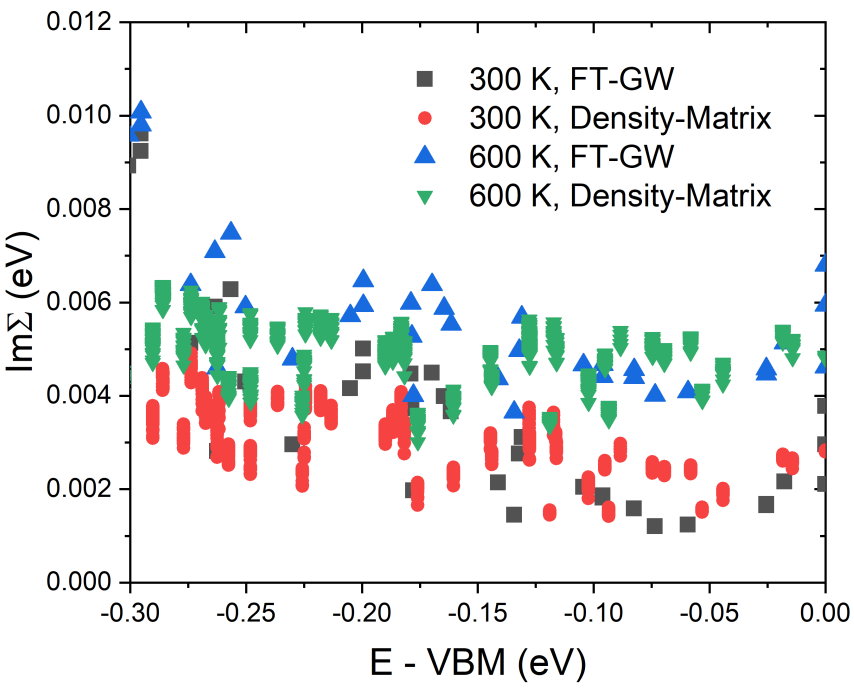

FIG. 10. $\operatorname{Im} \Sigma$ due to e-e scattering of valence electrons of $\mathrm{p}-$ type silicon computed by Eq. 46 (Density-Matrix) compared with those calculated by the finite-temperature GW method $(\mathrm{FT}-\mathrm{GW})^{97}$. $\mu$ is set to $0.05 \mathrm{eV}$ lower than Valence Band Maximum (VBM). For simplicity, SOC is not considered in this test.

Using Eq. 44, we have calculated the e-ph scattering rates and they are in good agreement with previous theoretical results ${ }^{96}$. For e-ph scattering, Eq. 44 will reproduce the imaginary part of the well-known Fan-Migdal self-energy 53 .

For e-i scattering, we have

$$
\frac{1}{\tau_{p, 1}^{\mathrm{e}-\mathrm{i}}}=\frac{2 \pi}{\hbar} n_{i} V_{\text {cell }} \sum_{2}\left|g_{12}^{i}\right|^{2} \delta_{\sigma}^{G}\left(\epsilon_{1}-\epsilon_{2}\right) .
$$

The above equation (Eq. 45) is consistent with Ref. 54,

For e-e scattering, neglecting the exchange contribution, which is a commonly-used approximation $\underline{50197}$,

$$
\frac{1}{\tau_{p, 1}^{\mathrm{e}-\mathrm{e}}}=\frac{2 \pi}{\hbar} \sum_{2 \neq 1,34}\left|A_{1324}\right|^{2}\left[\begin{array}{c}
f_{2}^{\mathrm{eq}} f_{4}^{\mathrm{eq}}\left(1-f_{3}^{\mathrm{eq}}\right)+ \\
\left(1-f_{2}^{\mathrm{eq}}\right) f_{3}^{\mathrm{eq}}\left(1-f_{4}^{\mathrm{eq}}\right)
\end{array}\right] .
$$

To verify our implementation of e-e scattering term, we have calculated $\operatorname{Im} \Sigma$ due to e-e scattering of valence electrons of p-type silicon based on the above equation and compare it with those calculated by the finitetemperature GW method from first-principles ${ }^{97}$ as implemented in JDFTx. ${ }^{60}$ The JDFTx implementation, in turn, has been benchmarked to reproduce the expected dependence with temperature and carrier energy, $\operatorname{Im} \Sigma^{\mathrm{e}-\mathrm{e}} \propto\left(\varepsilon-\varepsilon_{F}\right)^{2}+\left(\pi k_{B} T\right)^{2}$, as expected for metals. ${ }^{73}$

From Fig. 10, we can see the results by two methods agree well for the energy range close to the Fermi level which is relevant to e-e scatterings due to energy conservation. This verifies our implementation of e-e scattering part. 


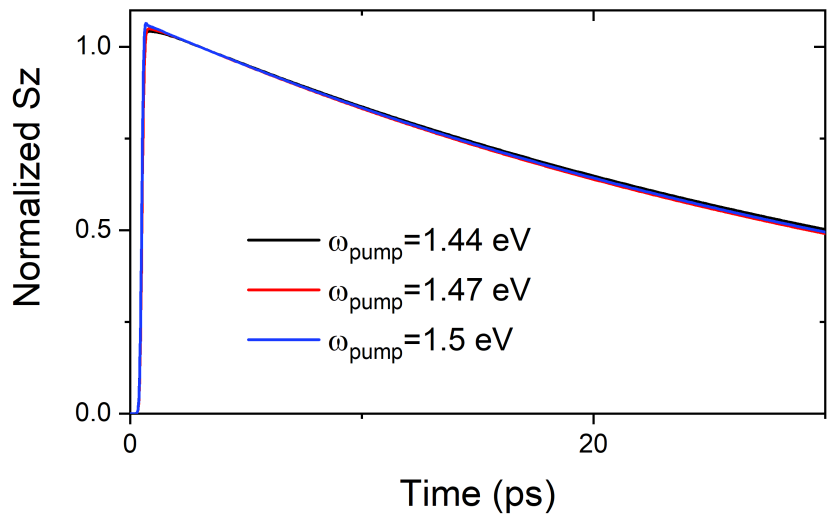

FIG. 11. $S_{z}(t)$ of $n$-GaAs with $n_{i}=2 \times 10^{16} \mathrm{~cm}^{-3}$ at 300 $\mathrm{K}$ with different pump pulse energies $\left(\omega_{\text {pump }}\right)$ varying with several $k_{B} T$.
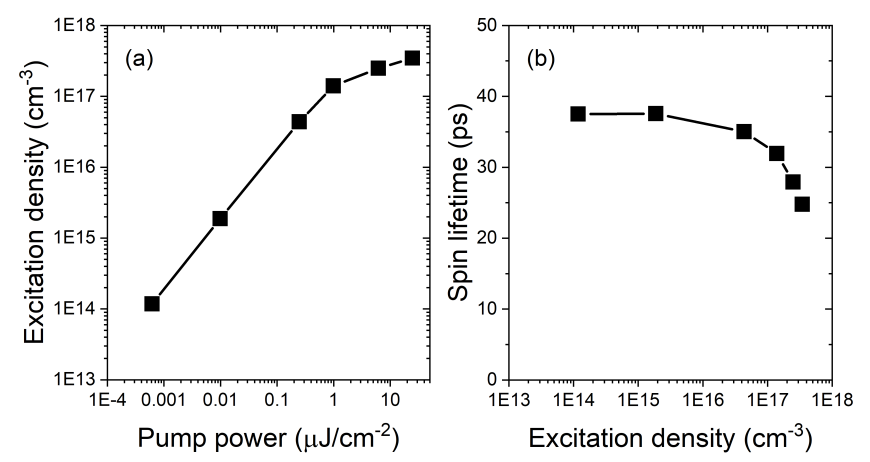

FIG. 12. (a) The excitation density as a function of the pump fluence (left panel) and (b) the spin lifetime as a function of the excitation density generated by a circularly polarized pump pulse for $n$-GaAs with $n_{i}=10^{14} \mathrm{~cm}^{-3}$ at $300 \mathrm{~K}$. $\omega_{\text {pump }}=1.47 \mathrm{eV}$.

\section{APPENDIX C: THE EFFECTS OF $\omega_{\text {pump }}$ AND PUMP FLUENCE ON SPIN RELAXATION OF GAAS AT $300 \mathrm{~K}$}

In Fig. 11, we study the $S_{z}$ relaxation dependence on pump-pulse energy changes with several $k_{B} T$. We can see that variation of $\omega_{\text {pump }}$ has very weak effects on spin dynamics of $n$-GaAs at $300 \mathrm{~K}$.

In Fig. $12 \mathrm{k}$, we study the effects of the pump fluence $I_{\text {pump }}$ on spin relaxation. Firstly, we can see that in the low pump fluence region or when $I_{\text {pump }}<1 \mu \mathrm{J} / \mathrm{cm}^{-2}$, the excitation density increases linearly with $I_{\text {pump }}$ but when $I_{\text {pump }}>1 \mu \mathrm{J} / \mathrm{cm}^{-2}$, the excitation density increases slower. This is because in high fluence cases, during the excitation by a pump pulse, a significant amount of conduction states have been already filled, which reduce the probability of the transitions from valence bands to con- duction bands. From Fig. 12 , we find that spin lifetime of $n$-GaAs decreases with the excitation density. This dependence may be explained based on the empirical DP relation ${ }^{2} \tau_{s, i} \sim \tau_{s, i}^{D P}=1 /\left[\bar{\tau}_{p} \cdot\left(\left\langle\boldsymbol{\Omega}^{2}\right\rangle-\left\langle\Omega_{i}^{2}\right\rangle\right)\right]$ as we discussed in Sec. IV A 2 . At $300 \mathrm{~K}$, generally $\left\langle\boldsymbol{\Omega}^{2}\right\rangle-\left\langle\Omega_{i}^{2}\right\rangle$ will increase with increasing free carrier density (through an increase of excitation density here), similar to what we find at $30 \mathrm{~K}$ shown in Fig. 55. On the other hand, $\bar{\tau}_{p}$ due to the electron-phonon scattering, which dominates carrier relaxation at $300 \mathrm{~K}$, is less sensitive to the variation of excitation density. Therefore, it is the increase of $\left\langle\Omega^{2}\right\rangle-\left\langle\Omega_{i}^{2}\right\rangle$ causing the decrease of spin lifetime when increasing excitation density.

\section{APPENDIX D: LAYER-RESOLVED QUANTITIES OF BILAYER WSE W $_{2}$}

For a bilayer with one layer above $z=0$ and another below, the operator $\hat{l}^{\text {top }}$ projecting any local quantity $A(\mathbf{r})$ to the top layer can be defined through the relation

$$
\hat{l}^{\mathrm{top}} A(\mathbf{r})=H(z) A(\mathbf{r}),
$$

where $z$ is the third component of $\mathbf{r}$ and $H(z)$ is Heaviside step function

$$
H(z)=\left\{\begin{array}{l}
1, z>0 \\
0, z \leq 0
\end{array}\right.
$$

Therefore, top layer hole occupation $f_{h}^{\text {top }}$ is

$$
\begin{aligned}
f_{h}^{\mathrm{top}} & =\operatorname{Tr}\left(\hat{l}^{\mathrm{top}}(1-f)\right) \\
& =\sum_{k n} l_{k, n n}^{\mathrm{top}}\left(1-f_{k n}\right),
\end{aligned}
$$

where $f$ is occupation and $l_{k, m n}^{\text {top }}=\left\langle k m\left|\hat{l}^{\text {top }}\right| k n\right\rangle=$ $\langle k m|H(z)| k n\rangle$. Tr means taking trace. $k$ is k-point index. $n$ and $m$ are band indices.

Moreover, top layer spin $s_{z}^{\text {top }}$ can be defined using the operator $\hat{l}^{\text {top }} \hat{s}_{z}$,

$$
\begin{aligned}
s_{z}^{\mathrm{top}} & =\operatorname{Tr}\left(\hat{l}^{\mathrm{top}} \hat{s}_{z} \hat{\rho}\right) \\
& =\sum_{k, l m n} l_{k, l m}^{\mathrm{top}} s_{z, k, m n} \rho_{k, n l} .
\end{aligned}
$$

Note that the commutator $\left[l^{\text {top }}, s_{z}\right]$ is found numerically close to zero for bilayer $\mathrm{WSe}_{2}$. This indicates that we can safely define $s_{z}^{\text {top }}$ as an observable using the above equations.
1 D. D. Awschalom, R. Hanson, J. Wrachtrup, and B. B. Zhou, "Quantum Technologies with Optically Interfaced
Solid-State Spins," Nat. Photon. 12, 516 (2018). 
2 I. Žutić, J. Fabian, and S. D. Sarma, "Spintronics: Fundamentals and Applications," Rev. Mod. Phys. 76, 323 (2004).

3 F. Meier and B. P. Zakharchenya, Optical Orientation (Elsevier, 2012).

${ }^{4}$ M. P. M. Dean, Y. Cao, X. Liu, S. Wall, D. Zhu, R. Mankowsky, V. Thampy, X. M. Chen, J. G. Vale, D. Casa, et al., "Ultrafast Energy-and MomentumResolved Dynamics of Magnetic Correlations in the PhotoDoped Mott Insulator Sr2IrO4," Nat. Mater. 15, 601-605 (2016).

5 A. Avsar, H. Ochoa, F. Guinea, B. Özyilmaz, B. J. van Wees, and I. J. Vera-Marun, "Colloquium: Spintronics in Graphene and Other Two-Dimensional Materials," Rev. Mod. Phys. 92, 021003 (2020).

6 J. J. Pla, K. Y. Tan, J. P. Dehollain, W. H. Lim, J. J. L. Morton, D. N. Jamieson, A. S. Dzurak, and A. Morello, "A Single-Atom Electron Spin Qubit in Silicon," Nature 489, 541-545 (2012).

7 J. Schliemann, "Colloquium: Persistent Spin Textures in Semiconductor Nanostructures," Rev. Mod. Phys. 89, 011001 (2017).

8 Dreyer, C. E. and Alkauskas, A. and Lyons, J. L. and Janotti, A. and Van de Walle, C. G., "First-Principles Calculations of Point Defects for Quantum Technologies," Annu. Rev. Mater. Res. 48, 1-26 (2018).

9 F. Wu, A. Galatas, R. Sundararaman, D. Rocca, and Y. Ping, "First-Principles Engineering of Charged Defects for Two-Dimensional Quantum Technologies," Phys. Rev. Mater. 1, 071001 (2017).

10 T. J. Smart, F. Wu, M. Govoni, and Y. Ping, "Fundamental Principles for Calculating Charged Defect Ionization Energies in Ultrathin Two-Dimensional Materials," Phys. Rev. Mater. 2, 124002 (2018).

11 F. Wu, T. J. Smart, J. Xu, and Y. Ping, "Carrier Recombination Mechanism at Defects in Wide Band Gap TwoDimensional Materials from First Principles," Phys. Rev. B 100, 081407 (2019).

12 F. Wu, D. Rocca, and Y. Ping, "Dimensionality and Anisotropicity Dependence of Radiative Recombination in Nanostructured Phosphorene," J. Mater. Chem. C 7, 12891-12897 (2019).

13 T. J. Smart, K. Li, J. Xu, and Y. Ping, "Intersystem Crossing and Exciton-Defect Coupling of Spin Defects in Hexagonal Boron Nitride," (2020), arXiv:2009.02830 [cond-mat.mtrl-sci].

${ }_{14}$ M. I. Dyakonov and V. I. Perel, "Spin Relaxation of Conduction Electrons in Noncentrosymmetric Semiconductors," Soviet Physics Solid State, Ussr 13, 3023-3026 (1972).

15 A. W. Cummings, J. H. Garcia, J. Fabian, and S. Roche, "Giant Spin Lifetime Anisotropy in Graphene Induced by Proximity Effects," Phys. Rev. Lett. 119, 206601 (2017).

16 D. Afanasiev, A. Gatilova, D. J. Groenendijk, B. A. Ivanov, M. Gibert, S. Gariglio, J. Mentink, J. Li, N. Dasari, M. Eckstein, et al., "Ultrafast Spin Dynamics in Photodoped Spin-Orbit Mott Insulator Sr2IrO4," Phys. Rev. X 9, 021020 (2019).

17 M. W. Wu, J. H. Jiang, and M. Q. Weng, "Spin Dynamics in Semiconductors," Phys. Rep. 493, 61-236 (2010).

18 R. Rosati, R. C. Iotti, F. Dolcini, and F. Rossi, "Derivation of Nonlinear Single-Particle Equations via Many-Body Lindblad Superoperators: A Density-Matrix Approach,"
Phys, Rev. B 90, 125140 (2014).

19 A. Habib, J. Xu, Y. Ping, and R. Sundararaman, "Electric Field and Substrate Effects Dominate Spin-Phonon Relaxation in Graphene," (2020), arXiv:2012.11550 [condmat.mtrl-sci].

${ }^{20}$ O. D. Restrepo and W. Windl, "Full First-Principles Theory of Spin Relaxation in Group-IV Materials," Phys. Rev. Lett. 109, 166604 (2012).

21 J. Park, J. Zhou, and M. Bernardi, "Spin-Phonon Relaxation Times in centrosymmetric Materials from First Principles," Phys, Rev. B 101, 045202 (2020).

22 D. V. Fedorov, M. Gradhand, S. Ostanin, I. V. Maznichenko, A. Ernst, J. Fabian, and I. Mertig, "Impact of Electron-Impurity Scattering on the Spin Relaxation Time in Graphene: A First-Principles Study," Phys. Rev. Lett. 110, 156602 (2013).

23 M. A. L. Marques, N. T. Maitra, F. M. S. Nogueira, E. K. U. Gross, and A. Rubio, Fundamentals of TimeDependent Density Functional Theory, Vol. 837 (Springer Science \& Business Media, 2012).

24 Z. Chen and L. Wang, "Role of Initial Magnetic Disorder: A Time-Dependent Ab Initio Study of Ultrafast Demagnetization Mechanisms," Sci. Adv. 5, eaau8000 (2019).

25 S. R. Acharya, V. Turkowski, G. P. Zhang, and T. S. Rahman, "Ultrafast Electron Correlations and Memory Effects at Work: Femtosecond Demagnetization in Ni," Phys. Rev. Lett. 125, 017202 (2020).

${ }^{26}$ K. Krieger, J. K. Dewhurst, P. Elliott, S. Sharma, and E. K. U. Gross, "Laser-Induced Demagnetization at Ultrashort Time Scales: Predictions of TDDFT," J. Chem. Theory Comput. 11, 4870-4874 (2015).

27 J. Xu, A. Habib, S. Kumar, F. Wu, R. Sundararaman, and Y. Ping, "Spin-Phonon Relaxation from a Universal Ab Initio Density-Matrix Approach," Nat. Commun. 11, $2780(2020)$.

28 J. M. Kikkawa and D. D. Awschalom, "Resonant Spin Amplification in n-Type GaAs," Phys. Rev. Lett. 80, 4313 (1998).

29 D. J. Hilton and C. L. Tang, "Optical Orientation and Femtosecond Relaxation of Spin-Polarized Holes in GaAs," Phys. Rev. Lett. 89, 146601 (2002).

30 J. H. Jiang and M. W. Wu, "Electron-Spin Relaxation in Bulk III-V Semiconductors from a Fully Microscopic Kinetic Spin Bloch Equation Approach," Phys, Rev. B 79, 125206 (2009).

31 A. Kamra and B. Ghosh, "The Role of Electron-Electron Scattering in Spin Transport," J. Appl. Phys 109, 024501 (2011).

32 F. Dettwiler, J. Fu, S. Mack, P. J. Weigele, J. C. Egues, D. D. Awschalom, and D. M. Zumbühl, "Stretchable Persistent Spin Helices in GaAs Quantum Wells," Phys. Rev. X 7, 031010 (2017).

33 D. Huber, M. Reindl, S. F. C. Da Silva, C. Schimpf, J. Martín-Sánchez, H. Huang, G. Piredda, J. Edlinger, A. Rastelli, and R. Trotta, "Strain-Tunable GaAs Quantum Dot: A Nearly Dephasing-Free Source of Entangled Photon Pairs on Demand," Phys. Rev. Lett. 121, 033902 (2018).

34 V. V. Belykh, A. Y. Kuntsevich, M. M. Glazov, K. V. Kavokin, D. R. Yakovlev, and M. Bayer, "Quantum Interference Controls the Electron Spin Dynamics in n-GaAs," Phys. Rev. X 8, 031021 (2018).

35 Y. Ohno, R. Terauchi, T. Adachi, F. Matsukura, and H. Ohno, "Spin Relaxation in GaAs (110) Quantum wells," 
Phys. Rev. Lett. 83, 4196 (1999).

36 A. V. Kimel, F. Bentivegna, V. N. Gridnev, V. V. Pavlov, R. V. Pisarev, and T. h. Rasing, "Room-Temperature Ultrafast Carrier and Spin Dynamics in GaAs Probed by the Photoinduced Magneto-Optical Kerr Effect," Phys, Rev. B 63, 235201 (2001).

37 P. E. Hohage, G. Bacher, D. Reuter, and A. D. Wieck, "Coherent Spin Oscillations in Bulk GaAs at Room Temperature," Appl. Phys. Lett. 89, 231101 (2006).

38 Z. G. Yu, S. Krishnamurthy, M. Van Schilfgaarde, and N. Newman, "Spin Relaxation of Electrons and Holes in Zinc-Blende Semiconductors," Phys, Rev. B 71, 245312 (2005).

39 M. D. Mower, G. Vignale, and I. V. Tokatly, "DyakonovPerel Spin Relaxation for Degenerate Electrons in the Electron-Hole Liquid," Phys, Rev. B 83, 155205 (2011).

40 G. Marchetti, M. Hodgson, J. McHugh, R. Chantrell, and I. D'Amico, "Spin Relaxation in GaAs: Importance of Electron-Electron Interactions," Materials 7, 2795-2814 (2014).

41 P. Dey, L. Yang, C. Robert, G. Wang, B. Urbaszek, X. Marie, and S. A. Crooker, "Gate-Controlled SpinValley Locking of Resident Carriers in WSe2 Monolayers," Phys. Rev. Lett. 119, 137401 (2017).

42 J. Li, M. Goryca, K. Yumigeta, H. Li, S. Tongay, and S. A. Crooker, "Valley relaxation of resident electrons and holes in a monolayer semiconductor: Dependence on carrier density and the role of substrate-induced disorder," Phys. Rev. Mater. 5, 044001 (2021).

43 Z. Gong, G. Liu, H. Yu, D. Xiao, X. Cui, X. Xu, and W. Yao, "Magnetoelectric effects and valley-controlled spin quantum gates in transition metal dichalcogenide bilayers," Nat. Commun. 4, 1-6 (2013).

${ }^{44} \mathrm{X} . \mathrm{Xu}, \mathrm{W}$. Yao, D. Xiao, and T. F. Heinz, "Spin and pseudospins in layered transition metal dichalcogenides," Nature Physics 10, 343-350 (2014).

45 H. Khani and S. P. Pishekloo, "Gate-controlled spinvalley-layer locking in bilayer transition-metal dichalcogenides," Nanoscale 12, 22281-22288 (2020).

46 X. Song, S. Xie, K. Kang, J. Park, and V. Sih, "Long-lived hole spin/valley polarization probed by Kerr rotation in monolayer WSe2," Nano Lett. 16, 5010-5014 (2016).

47 M. H. D. Guimaraes and B. Koopmans, "Spin accumulation and dynamics in inversion-symmetric van der Waals crystals," Phys. Rev. Lett. 120, 266801 (2018).

48 J. Ye, Y. Li, T. Yan, G. Zhai, and X. Zhang, "Ultrafast dynamics of spin generation and relaxation in layered WSe2," J. Phys. Chem. Lett. 10, 2963-2970 (2019).

49 R. Bertoni, C. W. Nicholson, L. Waldecker, H. Hübener, C. Monney, U. De Giovannini, M. Puppin, M. Hoesch, E. Springate, R. T. Chapman, et al., "Generation and evolution of spin-, valley-, and layer-polarized excited carriers in inversion-symmetric WSe2," Phys. Rev. Lett. 117, 277201 (2016).

${ }^{50}$ F. Rossi and T. Kuhn, "Theory of Ultrafast Phenomena in Photoexcited Semiconductors," Rev. Mod. Phys. 74, 895 (2002).

51 L. Yang, N. A. Sinitsyn, W. Chen, J. Yuan, J. Zhang, J. Lou, and S. A. Crooker, "Long-Lived Nanosecond Spin Relaxation and Spin Coherence of Electrons in Monolayer MoS2 and WS2," Nat. Phys. 11, 830-834 (2015).

52 R. C. Iotti and F. Rossi, "Phonon-induced dissipation and decoherence in solid-state quantum devices: Markovian versus non-Markovian treatments," Eur. Phys. J. B 90,
$1-14(2017)$

53 F. Giustino, "Electron-Phonon Interactions from First Principles," Rev. Mod. Phys. 89, 015003 (2017).

${ }^{54}$ C. Jacoboni, Theory of Electron Transport in Semiconductors: A Pathway from Elementary Physics to Nonequilibrium Green Functions, Vol. 165 (Springer Science \& Business Media, 2010).

55 Y. Joly, "Interaction matter-polarized light," (2009).

56 M. D'Alessandro and D. Sangalli, "Real-Time Modeling of Optical Orientation in GaAs: Generation and Decay of the Degree of Spin Polarization," Phys, Rev. B 102, 104437 (2020).

57 K. Hannewald, S. Glutsch, and F. Bechstedt, "QuantumKinetic Study of Femtosecond Pump-and-Probe Spectra of Bulk GaAs," Phys. Rev. B 61, 10792 (2000).

58 A. Molina-Sánchez, D. Sangalli, L. Wirtz, and A. Marini, "Ab Initio Calculations of Ultrashort Carrier Dynamics in Two-Dimensional Materials: Valley Depolarization in Single-Layer WSe2," Nano Lett. 17, 4549-4555 (2017).

59 N. Mainkar, D. A. Browne, and J. Callaway, "FirstPrinciples LCGO Calculation of the Magneto-Optical Properties of Nickel and Iron," Phys, Rev. B 53, 3692 (1996).

60 R. Sundararaman, K. Letchworth-Weaver, K. A. Schwarz, D. Gunceler, Y. Ozhabes, and T. A. Arias, "JDFTx: Software for Joint Density-Functional Theory," SoftwareX 6, 278-284 (2017).

61 O. Madelung, U. Rosseler, and M. Schulz, Group IV Elements, IV-IV and III-V Compounds. Part a - Lattice Properties (Springer-Verlag Berlin Heidelberg, 2001).

62 J. Sun, A. Ruzsinszky, and J. P. Perdew, "Strongly Constrained and Appropriately Normed Semilocal Density Functional," Phys. Rev. Lett. 115, 036402 (2015).

63 "See Supplemental Material at [url] for electronic band structure, phonon dispersions, convergence tests, and the treatment of scattering processes for holes.".

64 O. Madelung, Semiconductors (Springer, 1987).

65 S. Grimme, "Semiempirical GGA-type density functional constructed with a long-range dispersion correction," J. Comput. Chem. 27, 1787-1799 (2006).

66 M. K. Agarwal and P. A. Wani, "Growth conditions and crystal structure parameters of layer compounds in the series Mo1-xWxSe2," Mater. Res. Bull. 14, 825-830 (1979).

67 D. R. Hamann, "Optimized Norm-Conserving Vanderbilt Pseudopotentials," Phys, Rev. B 88, 085117 (2013).

68 N. Marzari and D. Vanderbilt, "Maximally Localized Generalized Wannier Functions for Composite Energy Bands," Phys. Rev. B 56, 12847 (1997).

69 A. M. Brown, R. Sundararaman, P. Narang, W. A. Goddard, and H. A. Atwater, "Nonradiative Plasmon Decay and Hot Carrier Dynamics: Effects of Phonons, Surfaces, and Geometry," ACS Nano 10, 957-966 (2016).

70 P. Narang, L. Zhao, S. Claybrook, and R. Sundararaman, "Effects of Interlayer Coupling on Hot Carrier Dynamics in Graphene-Derived van der Waals Heterostructures," Adv. Opt. Mater. 5, 1600914 (2017).

71 A. M. Brown, R. Sundararaman, P. Narang, A. M. Schwartzberg, W. A. Goddard III, and H. A. Atwater, "Experimental and Ab Initio Ultrafast Carrier Dynamics in Plasmonic Nanoparticles," Phys. Rev. Lett. 118, 087401 (2017).

72 A. Habib, R. Florio, and R. Sundararaman, "Hot Carrier Dynamics in Plasmonic Transition Metal Nitrides," J. Opt. 20, 064001 (2018). 
73 A. M. Brown, R. Sundararaman, P. Narang, W. A. Goddard III, and H. A. Atwater, "Ab Initio Phonon Coupling and Optical Response of Hot Electrons in Plasmonic Metals," Phys. Rev. B 94, 075120 (2016).

74 C. Verdi and F. Giustino, "Fröhlich Electron-Phonon Vertex from First Principles," Phys. Rev. Lett. 115, 176401 (2015).

75 T. Sohier, M. Calandra, and F. Mauri, "Two-dimensional Fröhlich interaction in transition-metal dichalcogenide monolayers: Theoretical modeling and first-principles calculations," Phys. Rev. B 94, 085415 (2016).

76 T. Sohier, M. Gibertini, M. Calandra, F. Mauri, and N. Marzari, "Breakdown of Optical Phonons' Splitting in Two-Dimensional Materials," Nano Lett. 17, 3758-3763 (2017).

77 P. Giannozzi, S. Baroni, N. Bonini, M. Calandra, R. Car, C. Cavazzoni, D. Ceresoli, G. L. Chiarotti, M. Cococcioni, I. Dabo, et al., "QUANTUM ESPRESSO: a Modular and Open-Source Software Project for Quantum Simulations of Materials," J. Condens. Matter Phys. 21, 395502 (2009).

78 A. R. Bungay, S. V. Popov, I. R. Shatwell, and N. I. Zheludev, "Direct Measurement of Carrier Spin Relaxation Times in Opaque Solids using the Specular Inverse Faraday Effect," Phys. Lett. A 234, 379-383 (1997).

79 G. Dresselhaus, "Spin-Orbit Coupling Effects in Zinc Blende Structures," Phys. Rev. 100, 580 (1955).

80 M. Goryca, N. P. Wilson, P. Dey, X. Xu, and S. A. Crooker, "Detection of thermodynamic "valley noise" in monolayer semiconductors: Access to intrinsic valley relaxation time scales," Sci. Adv. 5, eaau4899 (2019).

81 T. Yan, S. Yang, D. Li, and X. Cui, "Long valley relaxation time of free carriers in monolayer WSe2," Phys. Rev. B 95, 241406 (2017).

82 D. Wang, D. Han, D. West, N. Chen, S. Xie, Wei Q. Tian, V. Meunier, S. Zhang, and X. Li, "Excitation to defectbound band edge states in two-dimensional semiconductors and its effect on carrier transport," Npj Comput. Mater. 5, 1-6 (2019)

83 D. Edelberg, D. Rhodes, A. Kerelsky, B. Kim, J. Wang, A. Zangiabadi, C. Kim, A. Abhinandan, J. Ardelean, M. Scully, et al., "Approaching the intrinsic limit in transition metal diselenides via point defect control," Nano Lett. 19, 4371-4379 (2019).

84 D. Rhodes, S. H. Chae, R. Ribeiro P., and J. Hone, "Disorder in van der Waals heterostructures of 2D materials," Nat. Mater. 18, 541-549 (2019).
85 M. Yankowitz, D. McKenzie, and B. J. LeRoy, "Local spectroscopic characterization of spin and layer polarization in WSe2," Phys. Rev. Lett. 115, 136803 (2015).

${ }^{86}$ H. Kim and H. J. Choi, "Thickness dependence of work function, ionization energy, and electron affinity of Mo and W dichalcogenides from DFT and GW calculations," Phys. Rev. B 103, 085404 (2021).

87 W. Zhao, R. M. Ribeiro, M. Toh, A. Carvalho, C. Kloc, A. H. Castro Neto, and G. Eda, "Origin of indirect optical transitions in few-layer MoS2, WS2, and WSe2," Nano Lett. 13, 5627-5634 (2013).

88 M. Palummo, M. Bernardi, and J. C. Grossman, "Exciton radiative lifetimes in two-dimensional transition metal dichalcogenides," Nano Lett. 15, 2794-2800 (2015).

89 A. J. Goodman, A. P. Willard, and W. A. Tisdale, "Exciton trapping is responsible for the long apparent lifetime in acid-treated MoS2," Phys. Rev. B 96, 121404 (2017).

90 J. Towns, T. Cockerill, M. Dahan, I. Foster, K. Gaither, A. Grimshaw, V. Hazlewood, S. Lathrop, D. Lifka, G. D. Peterson, R. Roskies, J. R. Scott, and N. Wilkins-Diehr, "XSEDE: Accelerating Scientific Discovery," Comput. Sci. Eng. 16, 62-74 (2014).

91 R. Sundararaman and Y. Ping, "First-principles electrostatic potentials for reliable alignment at interfaces and defects," J. Chem. Phys. 146, 104109 (2017).

92 PI Tamborenea, MA Kuroda, and FL Bottesi, "Spin relaxation in n-doped GaAs due to impurity and electronelectron Elliot-Yafet scattering," Phys. Rev. B 68, 245205 (2003).

93 F. Aryasetiawan and S. Biermann, "Generalized Hedin's Equations for Quantum Many-Body Systems with SpinDependent Interactions," Phys. Rev. Lett. 100, 116402 (2008).

94 C. Grimaldi and P. Fulde, "Theory of screening of the phonon-modulated spin-orbit interaction in metals," Phys. Rev. B 55, 15523 (1997).

95 X. Wu, D. Vanderbilt, and D. R. Hamann, "Systematic Treatment of Displacements, Strains, and Electric Fields in Density-Functional Perturbation Theory," Phys, Rev. B 72, 035105 (2005).

96 J. Zhou and M. Bernardi, "Ab Initio Electron Mobility and Polar Phonon Scattering in GaAs," Phys. Rev. B 94, 201201 (2016).

97 L. X. Benedict, C. D. Spataru, and S. G. Louie, "Quasiparticle Properties of a Simple Metal at High Electron Temperatures," Phys, Rev. B 66, 085116 (2002). 